Rev. Mat. Iberoamericana 27 (2011), no. 1, 1-38

\title{
Global existence for the primitive equations with small anisotropic viscosity
}

Frédéric Charve and Van-Sang Ngo

\begin{abstract}
In this paper, we consider the primitive equations with zero vertical viscosity, zero vertical thermal diffusivity, and the horizontal viscosity and horizontal thermal diffusivity of size $\varepsilon^{\alpha}$ where $0<\alpha<\alpha_{0}$. We prove the global existence of a unique strong solution for large data provided that the Rossby number is small enough (the rotation and the vertical stratification are large).
\end{abstract}

\section{Introduction}

In this paper, we consider the primitive equations with no vertical viscosity and no vertical thermal diffusivity and we also suppose that the horizontal viscosity and thermal diffusivity go to zero when the rotation goes to infinity. We prove the convergence towards the quasi-geostrophic system and the global existence of a unique strong solution when the rotation is fast enough.

The primitive equations describe the hydrodynamical flow in a large scale (of order of hundreds or thousands of kilometers) on the earth, typically an ocean or the atmosphere, under the assumptions that vertical motion is much smaller than horizontal motion and that the fluid layer depth is small compared to the radius of the earth. Concerning the difference between the vertical and horizontal scales, it is also observed that for geophysical fluids, the vertical component of the diffusion term (viscosity or thermal diffusivity in the case of primitive equations) is much smaller than the horizontal component. In the case of a rotating fluid between two planes for example (see [26] and [16]), the viscosity has the form $\left(-\nu_{h} \Delta_{h}-\beta \varepsilon \partial_{3}^{2}\right)$. It is then relevant to consider a zero vertical diffusivity in the primitive equations.

2000 Mathematics Subject Classification: 35Q35, 35S30, 76D05, 76 U05.

Keywords: Primitive equations, quasi-geostrophic system, anisotropy, dispersion, Strichartz estimates. 
In the studies of the fluids of that scale, two important phenomena have to be considered: the earth rotation and the vertical stratification of the density due to the gravity. When the movement is observed in a frame located at the surface of the Earth, the rotation of the earth, defined by the Rossby number Ro, induces two additional force terms in the equations: the Coriolis force and the centrifugal force. The latter is included in the gravity gradient term in the right-hand side of the equations and has no important influence in our work. The Coriolis force, on the other hand, induces a vertical rigidity in the fluid. Under a fast rotation, the velocity of each particle of an homogeneous fluid, which has the same horizontal coordinates, is the same. This is called the phenomenon of Taylor-Proudman columns.

Gravity forces the fluid masses to have a vertical structure: heavier layers lay under lighter ones. Internal movements in the fluid tend to destroy this structure and the gravity basically tries to restore it, which gives a horizontal rigidity (to be opposed to the vertical rigidity induced by the rotation). In order to formally estimate the importance of this rigidity, we also compare the typical time scale of the system with the Brunt-Väisälä frequency and define the Froude number Fr. We will not give more details here and, for more physical considerations, we refer to [17], [18], [39], and [6] for example.

The primitive equations are obtained with moment, energy, mass conservations and scale simplifications by choosing the same scale for the rotation and stratification (see Embid and Majda [21]). In what follows, we denote by $\varepsilon$ the Rossby number and we set $F r=\varepsilon F$, where $F r$ is the Froude number, and $\varepsilon>0$ will go to zero. In the following, we call $\varepsilon$ and $F$ the Rossby and Froude numbers. Then, the anisotropic primitive equations are give by

$$
\left(A P E_{\varepsilon}\right) \begin{cases}\partial_{t} v_{\varepsilon}^{1}+v_{\varepsilon} \cdot \nabla v_{\varepsilon}^{1}-\nu \Delta_{h} v_{\varepsilon}^{1}-\frac{1}{\varepsilon} v_{\varepsilon}^{2}=-\frac{\partial_{1} \Phi_{\varepsilon}}{\varepsilon} & \text { in } \mathbb{R}_{+} \times \mathbb{R}^{3} \\ \partial_{t} v_{\varepsilon}^{2}+v_{\varepsilon} \cdot \nabla v_{\varepsilon}^{2}-\nu \Delta_{h} v_{\varepsilon}^{2}+\frac{1}{\varepsilon} v_{\varepsilon}^{1}=-\frac{\partial_{2} \Phi_{\varepsilon}}{\varepsilon} & \text { in } \mathbb{R}_{+} \times \mathbb{R}^{3} \\ \partial_{t} v_{\varepsilon}^{3}+v_{\varepsilon} \cdot \nabla v_{\varepsilon}^{3}-\nu \Delta_{h} v_{\varepsilon}^{3}+\frac{1}{F \varepsilon} \theta_{\varepsilon}=-\frac{\partial_{3} \Phi_{\varepsilon}}{\varepsilon} & \text { in } \mathbb{R}_{+} \times \mathbb{R}^{3} \\ \partial_{t} \theta_{\varepsilon}+v_{\varepsilon} \cdot \nabla \theta_{\varepsilon}-\nu^{\prime} \Delta_{h} \theta_{\varepsilon}-\frac{1}{F \varepsilon} v_{\varepsilon}^{3}=0 & \text { in } \mathbb{R}_{+} \times \mathbb{R}^{3} \\ \operatorname{div} v_{\varepsilon}=0 & \text { in } \mathbb{R}_{+} \times \mathbb{R}^{3} \\ \left(v_{\varepsilon}, \theta_{\varepsilon}\right)_{\left.\right|_{t=0}}=\left(v_{0}, \theta_{0}\right) & \text { in } \mathbb{R}^{3},\end{cases}
$$

where $\Delta_{h}=\partial_{x_{1}}^{2}+\partial_{x_{2}}^{2}$ is the horizontal Laplacian, and where $\nu$ and $\nu^{\prime}$ are the horizontal viscosity and the horizontal thermal diffusivity, which are of order $\varepsilon^{\alpha}$, where $\alpha$ is a positive constant which will be made more precise in the last section. The vector field $v_{\varepsilon}(t, x)$ is the fluid velocity, $\theta_{\varepsilon}(t, x)$ is the (scalar) density fluctuation and $\Phi_{\varepsilon}(t, x)$ is the geopotential 
term, containing the pressure and the centrifugal force. Notice that, in the case of meteorology problems, $\theta_{\varepsilon}$ is only a function of the temperature whereas, in oceanography, $\theta_{\varepsilon}$ depends on both temperature and salinity. For more details on the physical meanings of the quantities, we refer the reader to [6], [18], [25] and [39].

Denoting $U_{\varepsilon}$ the pair $\left(v_{\varepsilon}, \theta_{\varepsilon}\right)$, we can write the system $\left(A P E_{\varepsilon}\right)$ in the more compact form

$\left(A P E_{\varepsilon}\right)$

$$
\left\{\begin{array}{l}
\partial_{t} U_{\varepsilon}+U_{\varepsilon} \cdot \nabla U_{\varepsilon}-L U_{\varepsilon}+\frac{1}{\varepsilon} \mathcal{A} U_{\varepsilon}=\frac{1}{\varepsilon}\left(-\nabla \Phi_{\varepsilon}, 0\right) \\
\operatorname{div} v_{\varepsilon}=0 \\
U_{\left.\varepsilon\right|_{t=0}}=U_{0, \varepsilon}
\end{array}\right.
$$

where

$$
U_{\varepsilon} \cdot \nabla U_{\varepsilon}=v_{\varepsilon} \cdot \nabla U_{\varepsilon}=\sum_{i=1}^{3} v_{\varepsilon}^{i} \cdot \partial_{i} U_{\varepsilon},
$$

the matrix $\mathcal{A}$ is defined by

$$
\mathcal{A}=\left(\begin{array}{cccc}
0 & -1 & 0 & 0 \\
1 & 0 & 0 & 0 \\
0 & 0 & 0 & F^{-1} \\
0 & 0 & -F^{-1} & 0
\end{array}\right)
$$

and $L$ denotes the linear operator

$$
L U_{\varepsilon}=\left(\nu \Delta_{h} v_{\varepsilon}, \nu^{\prime} \Delta_{h} \theta_{\varepsilon}\right) .
$$

We introduce the potential vorticity:

$$
\Omega\left(U_{\varepsilon}\right) \stackrel{\text { def }}{=} \partial_{1} v_{\varepsilon}^{2}-\partial_{2} v_{\varepsilon}^{1}-F \partial_{3} \theta_{\varepsilon} .
$$

Then we define the orthogonal decomposition of $U_{\varepsilon}$ into its quasi-geostrophic part, and its oscillating part :

$$
U_{\varepsilon}=U_{\varepsilon, Q G}+\left(U_{\varepsilon}-U_{\varepsilon, Q G}\right) \stackrel{\text { def }}{=} U_{\varepsilon, Q G}+U_{\varepsilon, o s c},
$$

where

$$
U_{\varepsilon, Q G} \stackrel{\text { def }}{=}\left(\begin{array}{c}
-\partial_{2} \Delta_{F}{ }^{-1} \Omega\left(U_{\varepsilon}\right) \\
\partial_{1} \Delta_{F}{ }^{-1} \Omega\left(U_{\varepsilon}\right) \\
0 \\
-F \partial_{3} \Delta_{F}{ }^{-1} \Omega\left(U_{\varepsilon}\right)
\end{array}\right), \quad U_{\varepsilon, \text { osc }} \stackrel{\text { def }}{=}\left(\begin{array}{c}
v_{\varepsilon}^{1}+\partial_{2} \Delta_{F}{ }^{-1} \Omega\left(U_{\varepsilon}\right) \\
v_{\varepsilon}^{2}-\partial_{1} \Delta_{F}{ }^{-1} \Omega\left(U_{\varepsilon}\right) \\
v_{\varepsilon}^{3} \\
\theta_{\varepsilon}+F \partial_{3} \Delta_{F}{ }^{-1} \Omega\left(U_{\varepsilon}\right)
\end{array}\right),
$$

and

$$
\Delta_{F} \stackrel{\text { def }}{=} \partial_{1}^{2}+\partial_{2}^{2}+F^{2} \partial_{3}^{3} .
$$

Remark 1.1 We refer to [8] to [10] for general properties of the potential vorticity, but we recall that $\Omega\left(U_{\varepsilon, o s c}\right)=0$ and $\Omega\left(U_{\varepsilon, Q G}\right)=\Omega\left(U_{\varepsilon}\right)$. 
In [39], assuming that the Brunt-Väisälä frequency is constant, the authors have shown that, when $\varepsilon$ goes to zero, the formal limit of the system $\left(A P E_{\varepsilon}\right)$ is the following quasi-geostrophic system:

$(Q G)$

$$
\left\{\begin{array}{l}
\widetilde{\partial_{t} \widetilde{U_{Q G}}}-\Gamma \widetilde{U_{Q G}}=-\left(\begin{array}{c}
-\partial_{2} \\
\partial_{1} \\
0 \\
-F \partial_{3}
\end{array}\right) \Delta_{F}^{-1}\left(\widetilde{U_{Q G}} \cdot \nabla \Omega\left(\widetilde{U_{Q G}}\right)\right) \\
\operatorname{div} \widetilde{U_{Q G}}=0 \\
\left.\widetilde{U_{Q G}}\right|_{t=0}=U_{0, Q G},
\end{array}\right.
$$

where $\widetilde{U_{Q G}} \cdot \nabla \stackrel{\text { def }}{=} \widetilde{v_{Q G}} \cdot \nabla, \Gamma$ is the operator:

$$
\Gamma \stackrel{\text { def }}{=} \Delta_{h} \Delta_{F}^{-1}\left(\nu \partial_{1}^{2}+\nu \partial_{2}^{2}+\nu^{\prime} F^{2} \partial_{3}^{2}\right)
$$

and $U_{0, Q G}$ is the quasi-geostrophic part of the initial data $U_{0}$.

In real observations, the rotation is usually not fast enough and the fluid not homogeneous enough, but this quasi-geostrophic system (which considers not only rotation terms but also vertical stratification terms) is still a very good approximation of the behavior of real geophysical fluids. From the mathematical point of view, the quasi-geostrophic system has an "almost" bidimensional behavior, thus, is globally wellposed. This fact is very useful in proving the global existence of solutions of the primitive equations when the rotation is fast enough.

Before stating the main theorem of this paper, let us briefly recall known results for the primitive equations. We emphasize that all the following mentioned results concern the isotropic case where the viscosity and the thermal diffusivity are fixed and are the same in all directions (that is $\nu \Delta_{h}$ and $\nu^{\prime} \Delta_{h}$ are replaced by $\nu \Delta$ and $\nu^{\prime} \Delta$ in the system $\left(A P E_{\varepsilon}\right)$ with constant $\nu$ and $\left.\nu^{\prime}\right)$.

First, we remark that, in the case of isotropic geophysical fluids, there are results corresponding to the Leray ([31]) and Fujita-Kato ([23]) theorems. The existence of a global weak solution and the existence and uniqueness of a local strong solution (which is global for small data) are known. We also remark that there are two different cases to be considered for the primitive equations in the whole space $\mathbb{R}^{3}$, namely the case where $F=1$ and where there is no dissipation with respect to $\varepsilon$ and the case where $F \neq 1$ and where the dissipation plays an important role. In [13], Chemin studied the first case and proved the convergence of the solutions towards those of the quasigeostrophic system for regular, well prepared data, under the assumption that $\left|\nu-\nu^{\prime}\right|$ is small. 
In the case where $F \neq 1$, the dissipation allows not only to prove the convergence of the primitive system towards the quasi-geostrophic system but also to prove the global existence of solutions when the Rossby number $\varepsilon$ is small (fast rotation). Thus, the quasi-geostrophic system can be interpreted as the asymptotics of the primitive equations when the rotation and stratification have an important influence. In this case, one needs to decompose the general solution into its quasi-geostrophic part (which has the same structure as the solution of the quasi-geostrophic system) and its oscillating part (the remaining terms). Then, filtering the fast oscillating terms leads to the stabilization the system and makes it tend to the slow quasi-geostrophic system. In [8], [9] and [10], in the case of constant isotropic viscosity and thermal diffusivity, the first author of this paper proved, for various initial data, that the limit system of the primitive equations is the quasi-geostrophic system $(Q G)$ when $\varepsilon$ goes to zero. In [7], he considered strong solutions and showed that the oscillating part goes to zero, whereas the quasi-geostrophic part goes to the unique global solution of the 3D quasi-geostrophic system whose initial data is the quasi-geostrophic part of the initial data. As in [21], [24] and [14], the methods used in [7] are given by the study of spectral properties of the matrix $-\widehat{L+\frac{1}{\varepsilon} \mathbb{P}} \mathcal{A}$, where $\mathbb{P}$ denotes the Leray projection of $\mathbf{L}^{2}$ onto the subspace of divergence free vector fields.

Finally, we recall some further results concerning the primitive equations. We refer to Lions, Temam and Wang ([32] and [33]), for the asymptotic expansion of the primitive equations with respect to the Rossby number $\varepsilon$, where one recovers the geostrophic system and the quasi-geostrophic system as expansions of zero and first order respectively. In [4], Beale and Bourgeois studied the quasi-geostrophic system in periodic domains and proved the convergence of the primitive system towards the quasi-geostrophic system when $\varepsilon$ goes to zero, provided that the initial data are well prepared in $\mathbf{H}^{3}$. This convergence was also proved by Gallagher in [24], using Schochet's methods. For the case of inviscid primitive equations with $F \neq 1$, in [29], Iftimie proved the local convergence in time of the primitive system towards the quasi-geostrophic system with regular data.

The anisotropic primitive equations with evanescent viscosity and evanescent thermal diffusivity introduce new difficulties. First of all, in the case where there is no vertical viscosity and thermal diffusivity, the theorems of Leray-type are no longer available and the existence of a weak solution is still open. For strong solutions, the problem comes from the lack of regularizing effect in the third direction. Here, the fundamental idea is to use the divergence free property of the velocity. We note that the vertical derivative in the term $u \cdot \nabla$ is multiplied by $u^{3}$. However, the divergence free condition implies that $\partial_{3} u^{3}=-\partial_{1} u^{1}-\partial_{2} u^{2}$, so we can still use the regularizing effect induced by the horizontal viscosity and thermal diffusivity. 
In order to state our results, we need to introduce anisotropic Sobolev spaces. In what follows, we use the index " $\mathrm{h}$ " (respectively "v" or " 3 ") to refer to the horizontal variable (respectively to the vertical variable). Thus, $x_{h}=\left(x_{1}, x_{2}\right)$ and $\xi_{h}=\left(\xi_{1}, \xi_{2}\right)$. The anisotropic Sobolev spaces $H^{\sigma, s}$ are defined as the closure of the set of smooth functions under the norm

$$
\|u\|_{H^{\sigma, s}} \stackrel{\text { def }}{=}\left(\int_{\mathbb{R}^{3}}\left(1+\left|\xi_{h}\right|^{2}\right)^{\sigma}\left(1+\left|\xi_{3}\right|^{2}\right)^{s}|\widehat{u}|^{2} d \xi\right)^{\frac{1}{2}} .
$$

In the spirit of [15], we can prove the following theorem for the local existence (global existence for small data) of a strong solution of the primitive equations. The proof of this theorem follows the lines of the proof of existence given in [15] in the case of anisotropic rotating fluids. The uniqueness is proved as in [30]. For these reasons, the details are left to the reader.

Theorem 1.2 (Local Existence) Let $s>\frac{1}{2}, \varepsilon>0$ and $U_{0}=\left(u_{0}, \theta_{0}\right)$ be a divergence-free vector field (i.e. div $u_{0}=0$ ) in $H^{0, s}$. Then there exists $T_{\varepsilon}>0$ such that the system $\left(A P E_{\varepsilon}\right)$ has a unique solution $U_{\varepsilon}$ in $L^{\infty}\left(\left[0, T_{\varepsilon}\right], H^{0, s}\right)$ and $\nabla_{h} U_{\varepsilon} \in L^{2}\left(\left[0, T_{\varepsilon}\right], H^{0, s}\right)$.

Moreover, there exists a constant $c>0$ such that if the initial data satisfies $\left\|U_{0}\right\|_{H^{0, s}} \leq c \min \left(\nu_{\varepsilon}, \nu_{\varepsilon}^{\prime}\right)$, then $T_{\varepsilon}=\infty$.

Proving the global existence of strong solutions for large data in this case is more delicate. To simplify the situation, we go back to a much simpler system, namely the system of rotating fluids:

$$
\left\{\begin{array}{l}
\partial_{t} u+u \cdot \nabla u-\nu \Delta_{h} u+\frac{1}{\varepsilon} u \wedge \mathrm{e}_{3}=-\nabla p \\
\operatorname{div} u=0 \\
u_{\left.\right|_{t=0}}=u_{0} .
\end{array}\right.
$$

Let $w_{F}$ be the solution of the linear free waves system corresponding to $(N S C)$

$$
\left\{\begin{array}{l}
\partial_{t} w_{F}-\nu \Delta_{h} w_{F}+\frac{1}{\varepsilon} w_{F} \wedge \mathrm{e}_{3}=-\nabla p_{F} \\
\operatorname{div} w_{F}=0 \\
w_{F \mid t=0}=w_{0} .
\end{array}\right.
$$

In [15], when the viscosity $\nu>0$ is fixed, Chemin, Desjardins, Gallagher and Grenier proved the following Strichartz-type estimates:

Theorem 1.3 For any $0<r<R$, there exists a constant $C_{r, R}>0$ such that if the support of the Fourier transform of $w_{0}$ is localized in the set $\left\{\xi \in \mathbb{R}^{3}|| \xi_{3} \mid \geq r\right.$ and $\left.|\xi| \leq R\right\}$, then, for any $1 \leq p \leq+\infty$, we have

$$
\left\|w_{F}\right\|_{\mathbf{L}^{p}\left([0, T], \mathbf{L}_{h}^{\infty} \mathbf{L}_{v}^{2}\left(\mathbb{R}^{3}\right)\right)} \leq C_{r, R} \varepsilon^{\frac{1}{4 p}}\left\|w_{0}\right\|_{\mathbf{L}^{2}\left(\mathbb{R}^{3}\right)} .
$$


Here, the anisotropic Lesbesgue spaces $L_{h}^{p} L_{v}^{q}$ with $p, q \geq 1$ are defined by the norm

$$
\|u\|_{L_{h}^{p} L_{v}^{q}}=\left(\int_{\mathbb{R}_{h}^{2}}\left(\int_{\mathbb{R}_{v}}\left|u\left(x_{h}, x_{3}\right)\right|^{q} d x_{3}\right)^{\frac{p}{q}} d x_{h}\right)^{\frac{1}{p}} .
$$

Then using the so-called "bootstrap" argument, they proved the global existence of a strong solution for large data, provided that $\varepsilon$ is small enough.

Now, if in the equations $(N S C)$, the viscosity $\nu$ is not fixed, but depends on $\varepsilon$, say $\nu=\varepsilon^{\alpha}$, with $\alpha>0$, an additional difficulty arises in showing global existence of strong solutions. Indeed, by using the method of [15], in the energy estimates, we have to deal with the coefficient $\frac{1}{\nu}=\frac{1}{\varepsilon^{\alpha}}$, which goes to infinity when $\varepsilon$ goes to 0 . Nevertheless, in [35], the second author of this paper proved the global existence of a unique strong solution of $(N S C)$ with $\nu=\varepsilon^{\alpha}$, for large data, when $\varepsilon$ is small and $\alpha \leq \alpha_{0}$. The idea is to carefully study the dependence with respect to the cut-off radii $r$ and $R$ of the constant $C_{r, R}$ arising in the Strichartz estimates in order to get an estimate which is able to absorb the blowing-up term $\frac{1}{\varepsilon^{\alpha}}$.

The goal of this paper is to extend the result obtained in [35] to the primitive equations $\left(A P E_{\varepsilon}\right)$ and to show global existence of strong solutions of $\left(A P E_{\varepsilon}\right)$ for large data when $\varepsilon$ is small enough, in the case where $\nu=\varepsilon^{\alpha}$, $\nu^{\prime}=\rho \varepsilon^{\alpha}$ for $\alpha \leq \alpha_{0}$ and without any particular assumption on $\rho$. We will adapt the computations of eigenvalues and eigenvectors of the linearized primitive equations developed by F.Charve in [8], [7], [9], and [10] in the isotropic case to the anisotropic case.

We remark that, if $\nu \sim \nu^{\prime} \sim \varepsilon^{\alpha}$, then unlike the case of rotating fluids ([35]) where the limit system is zero, the limit system of the primitive equations, when $\varepsilon$ goes to zero, is the inviscid quasi-geostrophic system, which has no regularizing effect. So we have to estimate the $\mathbf{H}^{\sigma}$-norm, with $\sigma>\frac{5}{2}$, of the solution of the quasi-geostrophic system. The theory of hyperbolic systems implies the existence of a unique strong solution of this quasi-geostrophic system in $\mathbf{H}^{\sigma}$ with $\sigma>\frac{5}{2}$, but this solution could exponentially blow up in time at infinity. We have two different cases:

- The quasi-geostrophic part of the initial data is zero: the limit system is then zero, so we can use the idea of [35] with the adapted computations from [7] and [9] to prove a Strichartz-type estimate which will help to absorb the blow-up term and then prove the global existence of strong solutions of $\left(A P E_{\varepsilon}\right)$ for large data when $\varepsilon$ is small. This is the case considered in this paper.

- The quasi-geostrophic part of the initial data is not zero: the limit system is then a hyperbolic-type system. Mathematically, an idea to deal with this kind of system is to add a "friction" term $\gamma u$, with $\gamma>0$, 
in order to obtain an exponential dissipation in time which will balance the possible exponential blow-up of the solution of the quasigeostrophic system. We will treat this case in a subsequent paper.

Before stating the main theorem of this paper, we recall that, for $\eta \in \mathbb{R}$, the usual homogenous Sobolev space $\dot{H}^{\eta}$ is defined by the (semi-)norm

$$
\|u\|_{\dot{H}^{\eta}}=\left(\int_{\mathbb{R}^{3}}|\xi|^{2 \eta}|\widehat{u}|^{2} d \xi\right)^{\frac{1}{2}} .
$$

For $s>\frac{1}{2}$ and $\eta>0$, we define

$$
Y_{s, \eta}=H^{\eta, s+\eta} \cap L_{h}^{2} \dot{H}_{v}^{-2 \eta} \cap \dot{H}_{h}^{-\eta} H_{v}^{s} .
$$

The main result of this paper is the following

Theorem 1.4 (Global Existence) Let $s>\frac{1}{2}$ and $\eta>0$. There exists $\alpha_{0}>0$ and for any $r_{0}>0$, a positive number $\varepsilon_{0}=\varepsilon_{0}\left(r_{0}\right)$, such that, for all $0<\alpha<\alpha_{0}$, for all $0<\varepsilon<\varepsilon_{0}$ and for all $U_{0} \in Y_{s, \eta}$ with a zero quasigeostrophic part and with $\left\|U_{0}\right\|_{Y_{s, \eta}} \leq r_{0}$, the system $\left(A P E_{\varepsilon}\right)$ has a unique global solution $U_{\varepsilon}$ in $\mathbf{C}_{b}\left(\mathbb{R}_{+}, H^{0, s}\right)$, with $\nabla_{h} U_{\varepsilon} \in L^{2}\left(\mathbb{R}_{+}, H^{0, s}\right)$.

The structure of the paper is as follows. In Section 2 we recall basic results on anisotropic spaces and Littlewood-Paley decomposition. Section 3 is devoted to the spectral properties of the linearized problem corresponding to the system $\left(A P E_{\varepsilon}\right)$. In Section 4 , we study the dispersive effect and prove Strichartz estimates that will be used in the proof of Theorem 1.4. Finally, Section 5 contains the proof of Theorem 1.4 and a discussion on the constant $\alpha_{0}$.

\section{Preliminaries}

\subsection{Anisotropic spaces}

We briefly recall some basic properties of the anisotropic spaces. We refer to [14], [38] and [35] for more details. First we recall that the anisotropic Lebesgue spaces $L_{h}^{p} L_{v}^{q}$ with $p, q \geq 1$ are defined as

$$
L_{h}^{p} L_{v}^{q}\left(\mathbb{R}^{3}\right)=\left\{u \in \mathcal{S}^{\prime}\left(\mathbb{R}^{3}\right) \mid\|u\|_{L_{h}^{p} L_{v}^{q}}<+\infty\right\},
$$

where

$$
\|u\|_{L_{h}^{p} L_{v}^{q}}=\left(\int_{\mathbb{R}_{h}^{2}}\left(\int_{\mathbb{R}_{v}}\left|u\left(x_{h}, x_{3}\right)\right|^{q} d x_{3}\right)^{\frac{p}{q}} d x_{h}\right)^{\frac{1}{p}} .
$$


The order of integration is important. Indeed, let $\left(X_{1}, d \mu_{1}\right),\left(X_{2}, d \mu_{2}\right)$ be measurable spaces, if $1 \leq p \leq q$ and if $u: X_{1} \times X_{2} \rightarrow \mathbb{R}$ is a function in $L^{p}\left(X_{1} ; L^{q}\left(X_{2}\right)\right)$, then $u \in L^{q}\left(X_{2} ; L^{p}\left(X_{1}\right)\right)$ and

$$
\|u\|_{L^{q}\left(X_{2} ; L^{p}\left(X_{1}\right)\right)} \leq\|u\|_{L^{p}\left(X_{1} ; L^{q}\left(X_{2}\right)\right)} .
$$

We first recall the definition of the dyadic localization operators in the Fourier space. Let $\psi$ be an even smooth function in $C_{0}^{\infty}(\mathbb{R})$ such that the support of $\psi$ is contained in the ball $B_{\mathbb{R}}\left(0, \frac{4}{3}\right)$ and $\psi$ equals to 1 on a neighbourhood of the ball $B_{\mathbb{R}}\left(0, \frac{3}{4}\right)$. Let

$$
\varphi(\xi)=\psi\left(\frac{\xi}{2}\right)-\psi(\xi)
$$

Clearly the support of $\varphi$ is contained in the ring $\left\{\xi \in \mathbb{R}: \frac{3}{4} \leq|\xi| \leq \frac{8}{3}\right\}, \varphi$ is identically equal to 1 on the $\operatorname{ring}\left\{\xi \in \mathbb{R}: \frac{4}{3} \leq|\xi| \leq \frac{3}{2}\right\}$ and

$$
\forall \xi \in \mathbb{R}, \quad \psi(\xi)+\sum_{j \in \mathbb{N}} \varphi\left(\frac{\xi}{2^{j}}\right)=1,
$$

Let $\mathcal{F}$ and $\mathcal{F}^{-1}$ be the Fourier and inverse Fourier transforms respectively. We will often use the notation $\widehat{u}=\mathcal{F} u$. We introduce the following frequency truncation operators.

Definition 2.1 For any tempered distribution u, we set

$$
\begin{aligned}
\Delta_{q}^{v} u & =\mathcal{F}^{-1}\left(\varphi\left(2^{-q}\left|\xi_{3}\right|\right) \widehat{u}(\xi)\right), \quad \forall q \in \mathbb{N} \\
\Delta_{-1}^{v} u & =\mathcal{F}^{-1}\left(\psi\left(\left|\xi_{3}\right|\right) \widehat{u}(\xi)\right) \\
\Delta_{q}^{v} u & =0, \quad \forall q \leq-2 \\
S_{q}^{v} u & =\sum_{q^{\prime} \leq q-1} \Delta_{q^{\prime}}^{v} u .
\end{aligned}
$$

We refer to [5] and [12] for a more detailed construction of the dyadic decomposition. By using this definition, we can decompose all tempered distributions with respect to the vertical frequencies as

$$
u=\sum_{q \geq-1} \Delta_{q}^{v} u .
$$

We denote $\mathcal{F}_{v}$ the Fourier transform in the vertical direction. In our arguments, we shall use the following vertical Bernstein inequality (for the proof, see [12], [28] and [37]). 
Lemma 2.2 (Vertical Bernstein Lemma) Let $0<r_{1}<r_{2}$. There exists a positive constant $C_{0}$ such that, for any $u \in \mathcal{S}^{\prime}\left(\mathbb{R}^{3}\right)$ for which the support of $\mathcal{F}_{v} u$ is contained in the domain $\mathbb{R}_{\xi_{h}}^{2} \times\left\{2^{q} r_{1} \leq\left|\xi_{3}\right| \leq 2^{q} r_{2}\right\}$, we have:

$$
2^{q k} C_{0}^{-k}\|u\|_{\mathbf{L}_{h}^{p_{1}} \mathbf{L}_{v}^{p_{2}}} \leq\left\|\partial_{3}^{k} u\right\|_{\mathbf{L}_{h}^{p_{1}} \mathbf{L}_{v}^{p_{2}}} \leq 2^{q k} C_{0}^{k}\|u\|_{\mathbf{L}_{h}^{p_{1}} \mathbf{L}_{v}^{p_{2}}}, \forall k \geq 0, \forall p_{1}, p_{2} \geq 1
$$

and

$$
\|u\|_{\mathbf{L}_{h}^{p_{1}} \mathbf{L}_{v}^{p_{3}}} \leq C_{0} 2^{q\left(\frac{1}{p_{2}}-\frac{1}{p_{3}}\right)}\|u\|_{\mathbf{L}_{h}^{p_{1}} \mathbf{L}_{v}^{p_{2}}}, \quad \forall p_{1} \geq 1, \quad \forall 1 \leq p_{2} \leq p_{3} .
$$

This lemma allow us to rewrite the norm of the anisotropic Sobolev spaces $\mathbf{H}^{0, s}$ in terms of dyadic sums in the vertical frequencies.

Lemma 2.3 Suppose that $u \in \mathbf{H}^{0, s}$ with $s \in \mathbb{R}$, then we have

$$
\|u\|_{\mathbf{H}^{0, s}} \sim\left(\sum_{q} 2^{2 q s}\left\|\Delta_{q}^{v} u\right\|_{\mathbf{L}^{2}}^{2}\right)^{\frac{1}{2}} .
$$

We also have the following relation between each vertical dyadic block of a function $u$ and its $\mathbf{H}^{0, s}$-norm.

Lemma 2.4 Suppose that $u$ belongs to $\mathbf{H}^{0, s}$, then there exists a squaresummable sequence of positive numbers $\left\{c_{q}(u)\right\}$ with $\sum_{q} c_{q}(u)^{2}=1$, such that

$$
\left\|\Delta_{q}^{v} u\right\|_{\mathbf{L}^{2}} \leq c_{q}(u) 2^{-q s}\|u\|_{\mathbf{H}^{0, s}} .
$$

Finally, we define the spaces $\widetilde{\mathbf{L}}^{p}\left(I, \mathbf{H}^{0, s}\right)$, where $p \geq 2$ and $I$ is an interval of $\mathbb{R}_{+}$, as the closure of the set of smooth vector-fields with respect to the norms

$$
\|u\|_{\widetilde{\mathbf{L}}^{p}\left(I, \mathbf{H}^{0, s}\right)}=\left(\sum_{q} 2^{2 q s}\left\|\Delta_{q}^{v} u\right\|_{\mathbf{L}^{p}\left(I, \mathbf{L}^{2}\right)}^{2}\right)^{\frac{1}{2}} .
$$

With this definition, it is easy to see that, for any $p \geq 2$, the norm of $\widetilde{\mathbf{L}}^{p}\left(\mathbb{R}_{+}, \mathbf{H}^{0, s}\right)$ is stronger than the usual norm of $\mathbf{L}^{p}\left(\mathbb{R}_{+}, \mathbf{H}^{0, s}\right)$. From the above definition, we can prove the following lemma which is similar to Lemma 2.4.

Lemma 2.5 Suppose that $u$ belongs to $\widetilde{\mathbf{L}}^{p}\left(I, \mathbf{H}^{0, s}\right)$ where $I$ is an interval of $\mathbb{R}_{+}$, then there exists a square-summable sequence of positive numbers $\left(c_{q}(u)\right)$, with $\sum_{q} c_{q}(u)^{2}=1$, such that

$$
\left\|\Delta_{q}^{v} u\right\|_{\mathbf{L}^{p}\left(I, \mathbf{L}^{2}\right)} \leq c_{q}(u) 2^{-q s}\|u\|_{\tilde{\mathbf{L}}^{p}\left(I, \mathbf{H}^{0, s}\right)} .
$$




\subsection{A priori estimates}

In what follows, we always denote by $\left(c_{q}\right)$ (respectively $\left(d_{q}\right)$ ) a squaresummable (respectively summable) sequence of positive constants (which can depend on several parameters), such that $\sum_{q} c_{q}^{2}=1$ (respectively $\sum_{q} d_{q}=1$ ). We recall that, using the above dyadic decomposition, J.-Y. Chemin, B. Desjardins, I. Gallagher and E. Grenier have proved the following estimates (see $[15])$.

Lemma 2.6 For any real number $s>\frac{1}{2}$, there exists a constant $C$ such that for any vector fields $u$ and $v$, where $u$ is divergence free,

$$
\begin{aligned}
\left|\left\langle\Delta_{q}^{v}(u . \nabla v) \mid \Delta_{q}^{v} v\right\rangle_{\mathbf{L}^{2}}\right| \leq & C d_{q} 2^{-2 q s}\left(\left\|\nabla_{h} u\right\|_{\mathbf{H}^{0, s}}\|v\|_{\mathbf{H}^{0, s}}\left\|\nabla_{h} v\right\|_{\mathbf{H}^{0, s}}\right. \\
& \left.+\|u\|_{\mathbf{H}^{0, s}}^{\frac{1}{2}}\left\|\nabla_{h} u\right\|_{\mathbf{H}^{0, s}}^{\frac{1}{2}}\|v\|_{\mathbf{H}^{0, s}}^{\frac{1}{2}}\left\|\nabla_{h} v\right\|_{\mathbf{H}^{0, s}}^{\frac{3}{2}}\right),
\end{aligned}
$$

where $\left(d_{q}\right)$ is a summable sequence of positive constants with $\sum_{q} d_{q}=1$.

We also recall the following lemma [see [37], Lemma 3.3].

Lemma 2.7 For any real numbers $m, n, l \geq 1$ such that $\frac{1}{m}=\frac{1}{n}+\frac{1}{l}$, there exists a constant $C>0$ such that, for any functions $u$, $v$, we have

$$
\left\|\left[\Delta_{q}^{v} ; u\right] v\right\|_{\mathbf{L}_{v}^{2} \mathbf{L}_{h}^{m}} \leq C 2^{-q}\left\|\partial_{3} u\right\|_{\mathbf{L}_{v}^{\infty} \mathbf{L}_{h}^{n}}\|v\|_{\mathbf{L}_{v}^{2} \mathbf{L}_{h}^{l}} .
$$

In the previous paragraph, we introduced the vertical truncation and recalled the vertical Bernstein inequalities to define the anisotropic Sobolev spaces. We will also need to define truncations and anisotropic Bernstein inequalities in all three directions.

Definition 2.8 For $0<r<R$, we introduce the set

$$
\mathcal{C}_{r, R}=\left\{\xi \in \mathbb{R}^{3}:\left|\xi_{h}\right| \geq r,\left|\xi_{3}\right| \geq r,|\xi| \leq R\right\} .
$$

Remark 2.9 In general, $r$ will be chosen very small and $R$ very large.

We have the following anisotropic Bernstein inequalities.

Lemma 2.10 (Anisotropic Bernstein Lemma) Let $0<r_{1}<r_{2}$. There exists a positive constant $C_{0}$ such that, for any $u \in \mathcal{S}^{\prime}\left(\mathbb{R}^{3}\right)$ for which the support of $\mathcal{F} u$ is contained in the set $2^{q} \mathcal{C}_{r_{1}, r_{2}}$, we have, $\forall k \geq 0, \forall p_{1}, p_{2} \geq 1$,

$$
2^{q k} C_{0}^{-k}\|u\|_{\mathbf{L}_{h}^{p_{1}} \mathbf{L}_{v}^{p_{2}}} \leq \sup _{|\alpha|=k}\left\|\partial^{\alpha} u\right\|_{\mathbf{L}_{h}^{p_{1}} \mathbf{L}_{v}^{p_{2}}} \leq 2^{q k} C_{0}^{k}\|u\|_{\mathbf{L}_{h}^{p_{1}} \mathbf{L}_{v}^{p_{2}}},
$$

and, $\forall 1 \leq p_{1} \leq q_{1}, \forall 1 \leq p_{2} \leq q_{2}$,

$$
\|u\|_{\mathbf{L}_{h}^{q_{1}} \mathbf{L}_{v}^{q_{2}}} \leq C_{0} 2^{q\left(\frac{2}{p_{1}}-\frac{2}{q_{1}}+\frac{1}{p_{2}}-\frac{1}{q_{2}}\right)}\|u\|_{\mathbf{L}_{h}^{p_{1}} \mathbf{L}_{v}^{p_{2}}} .
$$




\section{Spectral aspects of the linear system}

The aim of this section is to determine the eigenvalues and eigenvectors of the matrix $\widehat{L-\frac{1}{\varepsilon} \mathbb{P}} \mathcal{A}$, and to establish the estimates of the associate projectors onto the eigenspaces. Recall that we already define

$$
\mathcal{C}_{r, R} \stackrel{\text { def }}{=}\left\{\xi \in \mathbb{R}^{3}:\left|\xi_{h}\right| \geq r,\left|\xi_{3}\right| \geq r,|\xi| \leq R\right\} .
$$

In what follows, we consider the following frequency cut-off function

$$
\Psi(\xi)=\chi\left(\frac{|\xi|}{R}\right)\left[1-\chi\left(\frac{2\left|\xi_{h}\right|}{r}\right)\right]\left[1-\chi\left(\frac{2\left|\xi_{3}\right|}{r}\right)\right],
$$

where $\chi$ is a $\mathcal{C}^{\infty}$-function from $\mathbb{R}$ to $\mathbb{R}$ such that

$$
\chi(x)=\left\{\begin{array}{lll}
1 & \text { if } & 0 \leq|x| \leq 1 \\
0 & \text { if } \quad|x| \geq 2
\end{array}\right.
$$

Thus, $\Psi \in \mathcal{D}\left(\mathbb{R}^{3}\right)$, supp $\Psi \subset \mathcal{C}_{\frac{r}{2}, 2 R}$ and $\Psi \equiv 1$ on $\mathcal{C}_{r, R}$. The frequency cut off operator is the following pseudo-differential operator:

$$
\mathcal{P}_{r, R} f \stackrel{\text { def }}{=} \mathcal{F}^{-1}(\Psi(\xi) \mathcal{F}(f)), \quad \forall f \in \mathcal{S}^{\prime},
$$

where $\mathcal{F}$ denotes the Fourier transform. In this paper, we do not consider a "fixed" frequency cut-off but a cut-off that depends on $\varepsilon$. So the frequency cut-off domain will be chosen as $\mathcal{C}_{r_{\varepsilon}, R_{\varepsilon}}$, with $R_{\varepsilon}=\varepsilon^{-\beta}$, and $r_{\varepsilon}=R_{\varepsilon}^{-\gamma}=\varepsilon^{\beta \gamma}$.

In this section, we consider the following cut-off linear system corresponding to $\left(A P E_{\varepsilon}\right)$

$$
\left\{\begin{array}{l}
\partial_{t} \bar{U}_{\varepsilon}-L \bar{U}_{\varepsilon}+\frac{1}{\varepsilon} \mathcal{A} \bar{U}_{\varepsilon}=\frac{1}{\varepsilon}\left(-\nabla \bar{\Phi}_{\varepsilon}, 0\right) \\
\operatorname{div} \bar{v}_{\varepsilon}=0 \\
\bar{U}_{\left.\varepsilon\right|_{t=0}}=\bar{U}_{0, \varepsilon}=\mathcal{P}_{r_{\varepsilon}, R_{\varepsilon}} U_{0, \varepsilon}
\end{array}\right.
$$

Applying the Leray projection $\mathbb{P}$ of $\mathbf{L}^{2}\left(\mathbb{R}^{3}\right)^{3}$ onto the subspace of divergencefree vector fields and then the Fourier transformation, we have

$$
\partial_{t} \widehat{\bar{U}}_{\varepsilon}=\mathbb{B}(\xi, \varepsilon) \widehat{\bar{U}_{\varepsilon}}
$$

where we define the matrix: $\mathbb{B}(\xi, \varepsilon)=\widehat{L-\frac{1}{\varepsilon} \mathbb{P}} \mathcal{A}=$

$$
=\left(\begin{array}{cccc}
-\nu_{\varepsilon}\left|\xi_{h}\right|^{2}+\frac{\xi_{1} \xi_{2}}{\varepsilon|\xi|^{2}} & \frac{\xi_{2}^{2}+\xi_{3}^{2}}{\varepsilon|\xi|^{2}} & 0 & \frac{\xi_{1} \xi_{3}}{\varepsilon F|\xi|^{2}} \\
-\frac{\xi_{1}^{2}+\xi_{3}^{2}}{\varepsilon|\xi|^{2}} & -\nu_{\varepsilon}\left|\xi_{h}\right|^{2}-\frac{\xi_{1} \xi_{2}}{\varepsilon|\xi|^{2}} & 0 & \frac{\xi_{2} \xi_{3}}{\varepsilon F|\xi|^{2}} \\
\frac{\xi_{2} \xi_{3}}{\varepsilon|\xi|^{2}} & -\frac{\xi_{1} \xi_{3}}{\varepsilon|\xi|^{2}} & -\nu_{\varepsilon}\left|\xi_{h}\right|^{2} & -\frac{\xi_{1}^{2}+\xi_{2}^{2}}{\varepsilon F|\xi|^{2}} \\
0 & 0 & \frac{1}{\varepsilon F} & -\nu_{\varepsilon}^{\prime}\left|\xi_{h}\right|^{2}
\end{array}\right) .
$$


The main result of this section is the following proposition, which will be used later in the proof of the Strichartz estimates

Proposition 3.1 There exists $\varepsilon_{0}>0$ such that for all $\varepsilon<\varepsilon_{0}$, for all $R_{\varepsilon}$ such that $\left|\nu_{\varepsilon}-\nu_{\varepsilon}^{\prime}\right| R_{\varepsilon}^{2} \varepsilon \ll 1$ and for all $\xi \in \mathcal{C}_{r_{\varepsilon}, R_{\varepsilon}}$ with $r_{\varepsilon}=R_{\varepsilon}^{-\gamma}$, the matrix $\mathbb{B}(\xi, \varepsilon)=\widehat{L-\frac{1}{\varepsilon} \mathbb{P}} \mathcal{A}$ is diagonalizable and its eigenvalues have the following asymptotic expansions with respect to $\varepsilon$ :

$$
\left\{\begin{array}{l}
\mu_{0}=-\nu_{\varepsilon}\left|\xi_{h}\right|^{2}, \\
\mu=-\left(\nu_{\varepsilon} \xi_{1}^{2}+\nu_{\varepsilon} \xi_{2}^{2}+\nu_{\varepsilon}^{\prime} F^{2} \xi_{3}^{2}\right) \frac{\left|\xi_{h}\right|^{2}}{|\xi|_{F}^{2}}+\varepsilon E_{2}, \\
\lambda=-\tau(\xi)\left|\xi_{h}\right|^{2}+i \frac{|\xi|_{F}}{\varepsilon F|\xi|}+\varepsilon E_{2}, \\
\bar{\lambda}=-\tau(\xi)\left|\xi_{h}\right|^{2}-i \frac{|\xi|_{F}}{\varepsilon F|\xi|}+\varepsilon E_{2},
\end{array}\right.
$$

where $|\xi|_{F}^{2}=\xi_{1}^{2}+\xi_{2}^{2}+F^{2} \xi_{3}^{3}, E_{2}$ is uniformly bounded on $\mathcal{C}_{r_{\varepsilon}, R_{\varepsilon}}$, and where:

$$
\varepsilon\left|E_{2}\right| \leq C_{F} \varepsilon\left|\nu_{\varepsilon}-\nu_{\varepsilon}^{\prime}\right| \cdot\left|\xi_{h}\right|^{2} \leq C_{F}\left|\nu_{\varepsilon}-\nu_{\varepsilon}^{\prime}\right| R_{\varepsilon}^{2} \varepsilon \ll 1,
$$

and

$$
\tau(\xi)=\frac{\nu_{\varepsilon}}{2}\left(1+\frac{F^{2} \xi_{3}^{2}}{|\xi|_{F}^{2}}\right)+\frac{\nu_{\varepsilon}^{\prime}}{2}\left(1-\frac{F^{2} \xi_{3}^{2}}{|\xi|_{F}^{2}}\right) \geq \min \left(\nu_{\varepsilon}, \nu_{\varepsilon}^{\prime}\right)>0 .
$$

Moreover, if we denote by $\mathcal{P}_{i}(\xi, \varepsilon)$, the projectors onto the eigenspaces corresponding to $\mu, \lambda$ and $\bar{\lambda}(i \in\{2,3,4\})$, and we set

$$
\mathbb{P}_{i}(u)=\mathcal{F}^{-1}\left(\mathcal{P}_{i}(\xi, \varepsilon)(\widehat{u}(\xi))\right),
$$

then for any divergence-free vector field $f$ whose Fourier transform is supported in $\mathcal{C}_{r_{\varepsilon}, R_{\varepsilon}}$, we have the following estimates:

$$
\left\|\mathbb{P}_{2} f\right\|_{H^{\sigma, s}} \leq \begin{cases}C_{F}\|f\|_{H^{\sigma, s}} & \text { if } \quad \Omega(f) \neq 0, \\ C_{F}\left|\nu_{\varepsilon}-\nu_{\varepsilon}^{\prime}\right| \varepsilon R_{\varepsilon}^{2}\|f\|_{H^{\sigma, s}} & \text { if } \quad \Omega(f)=0,\end{cases}
$$

and for $i=3,4$,

$$
\left\|\mathbb{P}_{i} f\right\|_{H^{\sigma, s}} \leq C_{F} R_{\varepsilon}^{1+\gamma}\|f\|_{H^{\sigma, s}} .
$$

Remark 3.2 If $R_{\varepsilon}=\varepsilon^{-\beta}, \nu_{\varepsilon}=\varepsilon^{\alpha}$ and $\nu_{\varepsilon}^{\prime}=\rho \varepsilon^{\alpha}$, then the condition

$$
\left|\nu_{\varepsilon}-\nu_{\varepsilon}^{\prime}\right| R_{\varepsilon}^{2} \varepsilon \ll 1, \quad \text { for small } \varepsilon>0,
$$

is equivalent to $1+\alpha-2 \beta>0$. 
In order to prove Proposition 3.1, we first determine the eigenvalues of $\mathbb{B}(\xi, \varepsilon)$ (the roots of its characteristic polynomial). One of the roots is easy to find. In order to calculate the others, we perform a change of variable on the remaining polynomial of degree 3 and then, we use the Cardan formulas. We show that, as $\varepsilon$ goes to zero, the discriminent is positive and we obtain the remaining three roots. The end of this section is devoted to the asymptotic expansions of these eigenvalues and to the proof of Proposition 3.1.

\subsection{Eigenvalues}

We recall that matrix $\mathbb{B}(\xi, \varepsilon)=\widehat{L-\frac{1}{\varepsilon} \mathbb{P}} \mathcal{A}$ writes:

$$
\mathbb{B}(\xi, \varepsilon)=\left(\begin{array}{cccc}
-\nu_{\varepsilon}\left|\xi_{h}\right|^{2}+\frac{\xi_{1} \xi_{2}}{\varepsilon|\xi|^{2}} & \frac{\xi_{2}^{2}+\xi_{3}^{2}}{\varepsilon|\xi|^{2}} & 0 & \frac{\xi_{1} \xi_{3}}{\varepsilon F|\xi|^{2}} \\
-\frac{\xi_{1}^{2}+\xi_{3}^{2}}{\varepsilon|\xi|^{2}} & -\nu_{\varepsilon}\left|\xi_{h}\right|^{2}-\frac{\xi_{1} \xi_{2}}{\varepsilon|\xi|^{2}} & 0 & \frac{\xi_{2} \xi_{3}}{\varepsilon F|\xi|^{2}} \\
\frac{\xi_{2} \xi_{3}}{\varepsilon|\xi|^{2}} & -\frac{\xi_{1} \xi_{3}}{\varepsilon|\xi|^{2}} & -\nu_{\varepsilon}\left|\xi_{h}\right|^{2} & -\frac{\xi_{1}^{2}+\xi_{2}^{2}}{\varepsilon F|\xi|^{2}} \\
0 & 0 & \frac{1}{\varepsilon F} & -\nu_{\varepsilon}^{\prime}\left|\xi_{h}\right|^{2}
\end{array}\right) .
$$

Its characteristic polynomial is:

$$
\chi_{\mathbb{B}}(X)=\left(X+\nu_{\varepsilon}\left|\xi_{h}\right|^{2}\right) P\left(X+\nu_{\varepsilon}\left|\xi_{h}\right|^{2}\right),
$$

with

$$
P(Y)=Y^{3}-\left(\nu_{\varepsilon}-\nu_{\varepsilon}^{\prime}\right)\left|\xi_{h}\right|^{2} Y^{2}+\frac{|\xi|_{F}^{2}}{\varepsilon^{2} F^{2}|\xi|^{2}} Y-\left(\nu_{\varepsilon}-\nu_{\varepsilon}^{\prime}\right) \frac{\xi_{3}^{2}\left|\xi_{h}\right|^{2}}{\varepsilon^{2}|\xi|^{2}}
$$

This polynomial has no particular property so, in order to find its roots, we use the Cardan formulas. We perform the following change of variable

$$
X=U-\frac{\nu_{\varepsilon}^{\prime}+2 \nu_{\varepsilon}}{3}|\xi|^{2}
$$

which takes into account the first change of variable (writing into the variable $\left(U+\nu_{\varepsilon}|\xi|^{2}\right)$ to simplify the expression of $\left.\chi_{\mathbb{B}}\right)$ and the one performed to reduce the polynomial into the particular formulation needed to apply the Cardan formulas $\left(U^{3}+p U+q\right)$. We refer to [8] and [9] for more details.

$$
P(X)=P\left(U+\frac{\nu_{\varepsilon}^{\prime}+2 \nu_{\varepsilon}}{3}|\xi|^{2}\right)=U^{3}+p U+q
$$


where

$$
\left\{\begin{array}{l}
p=\frac{|\xi|_{F}^{2}}{\varepsilon^{2} F^{2}|\xi|^{2}}-\frac{\left(\nu_{\varepsilon}-\nu_{\varepsilon}^{\prime}\right)^{2}}{3}\left|\xi_{h}\right|^{4}, \\
q=\left(\nu_{\varepsilon}-\nu_{\varepsilon}^{\prime}\right) \frac{\left|\xi_{h}\right|^{2}}{\varepsilon^{2}|\xi|^{2}}\left(\frac{|\xi|_{F}^{2}}{3 F^{2}}-\xi_{3}^{2}\right)-\frac{2}{27}\left(\nu_{\varepsilon}-\nu_{\varepsilon}^{\prime}\right)^{3}\left|\xi_{h}\right|^{6} .
\end{array}\right.
$$

Before applying the Cardan formulas we have to define the discriminant of the equation

$$
D=\frac{q^{2}}{4}+\frac{p^{3}}{27}
$$

We will see later that even if the radii $r_{\varepsilon}$ and $R_{\varepsilon}$ of $\mathcal{C}_{r_{\varepsilon}, R_{\varepsilon}}$ depend on the Rossby number, in the expression of $D$ every term is negligible compared to $\frac{|\xi|_{F}^{2}}{\varepsilon^{2} F^{2}|\xi|^{2}}$. Thus, in the following (the proof and precise asymptotic expansions are given in Section 3.3), if $\varepsilon$ is small enough, the discriminant is positive, so we can use the same methods as in [8] and [9] where $r$ and $R$ were fixed.

Now, as $D>0$ we can use the Cardan formulas to compute the eigenvalues of $\mathbb{B}(\xi, \varepsilon)$. We define the following quantities:

$$
\omega_{1}=\left(-\frac{q}{2}+D^{\frac{1}{2}}\right)^{\frac{1}{3}} \text { and } \omega_{2}=\left(-\frac{q}{2}-D^{\frac{1}{2}}\right)^{\frac{1}{3}} .
$$

Returning to the original variable, we obtain the eigenvalues of $\mathbb{B}(\xi, \varepsilon)$ :

$$
\left\{\begin{aligned}
\mu_{0} & =-\nu_{\varepsilon}\left|\xi_{h}\right|^{2} \\
\mu & =-\frac{\nu_{\varepsilon}^{\prime}+2 \nu_{\varepsilon}}{3}\left|\xi_{h}\right|^{2}+\omega_{1}+\omega_{2}, \\
\lambda & =-\frac{\nu_{\varepsilon}^{\prime}+2 \nu_{\varepsilon}}{3}\left|\xi_{h}\right|^{2}+\omega_{1} j+\omega_{2} j^{2} \\
\bar{\lambda} & =-\frac{\nu_{\varepsilon}^{\prime}+2 \nu_{\varepsilon}}{3}\left|\xi_{h}\right|^{2}+\omega_{1} j^{2}+\omega_{2} j
\end{aligned}\right.
$$

where $j$ is a primitive cube root of 1 .

\subsection{Eigenvectors}

In this paragraph, we provide some properties of the eigenvectors of $\mathbb{B}(\xi, \varepsilon)$. We refer to [8] and [9] for the precise computations. We use here the same methods and notations (except the changes induced by the horizontal Laplacian). Let

$$
\left\{\begin{array}{l}
A=\mu+\nu_{\varepsilon}\left|\xi_{h}\right|^{2}=\frac{\nu_{\varepsilon}-\nu_{\varepsilon}^{\prime}}{3}\left|\xi_{h}\right|^{2}+\omega_{1}+\omega_{2}, \\
B=\lambda+\nu_{\varepsilon}\left|\xi_{h}\right|^{2}=\frac{\nu_{\varepsilon}-\nu_{\varepsilon}^{\prime}}{3}\left|\xi_{h}\right|^{2}+\omega_{1}+\omega_{2} .
\end{array}\right.
$$


Since $A, B$ and $\bar{B}$ are the roots of the characteristic polynomial $P$ of $\mathbb{B}(\xi, \varepsilon)$ (see (3.8)), we have the following root-coefficient relations:

$$
\begin{cases}A B \bar{B} & =\left(\nu_{\varepsilon}-\nu_{\varepsilon}^{\prime}\right) \frac{\xi_{3}^{2}\left|\xi_{h}\right|^{2}}{\varepsilon^{2}|\xi|^{2}} \\ A+B+\bar{B} & =\left(\nu_{\varepsilon}-\nu_{\varepsilon}^{\prime}\right)\left|\xi_{h}\right|^{2} \\ A B+A \bar{B}+B \bar{B} & =\frac{|\xi|_{F}^{2}}{\varepsilon^{2} F^{2}|\xi|^{2}} .\end{cases}
$$

Let $W_{i}, i=2,3,4$, be the eigenvectors corresponding to the eigenvalues $\mu$, $\lambda, \bar{\lambda}$. Using the above relations, and after computations close to those in [8], we obtain the following matrix of change of basis: $Q=$

$$
\left(\begin{array}{crrr}
\xi_{2} \xi_{3} & \xi_{3}\left(\varepsilon \xi_{1} A+\xi_{2}\right) & \xi_{3}\left(\varepsilon \xi_{1} B+\xi_{2}\right) & \xi_{3}\left(\varepsilon \xi_{1} \bar{B}+\xi_{2}\right) \\
-\xi_{1} \xi_{3} & \xi_{3}\left(\varepsilon \xi_{2} A-\xi_{1}\right) & \xi_{3}\left(\varepsilon \xi_{2} B-\xi_{1}\right) & \xi_{3}\left(\varepsilon \xi_{2} \bar{B}-\xi_{1}\right) \\
-\varepsilon F^{2}\left(\nu_{\varepsilon}-\nu_{\varepsilon}^{\prime}\right)\left|\xi_{h}\right|^{2} \xi_{3}^{2} & -\varepsilon A\left|\xi_{h}\right|^{2} & -\varepsilon B\left|\xi_{h}\right|^{2} & -\varepsilon \bar{B}\left|\xi_{h}\right|^{2} \\
F \xi_{3}^{2} & F\left(\varepsilon^{2}|\xi|^{2} A^{2}+\xi_{3}^{2}\right) & F\left(\varepsilon^{2}|\xi|^{2} B^{2}+\xi_{3}^{2}\right) & F\left(\varepsilon^{2}|\xi|^{2} \bar{B}^{2}+\xi_{3}^{2}\right)
\end{array}\right) .
$$

We recall that the eigenvectors $W_{i}, i=2,3,4$, are orthogonal to $\left(\xi_{1}, \xi_{2}, \xi_{3}, 0\right)$ and form a basis of the Fourier subspace of all divergence-free vector fields. Thus, if a vector $f$, orthogonal to $\left(\xi_{1}, \xi_{2}, \xi_{3}, 0\right)$ in the space of frequencies, is written

$$
f=K_{2} W_{2}+K_{3} W_{3}+K_{4} W_{4}
$$

then

$$
\left(\begin{array}{c}
0 \\
K_{2} \\
K_{3} \\
K_{4}
\end{array}\right)=Q^{-1} f
$$

\section{Remark 3.3}

- The fact that $A, B$ and $\bar{B}$ are the roots of polynomial $P$ implies that we have the relation

$$
\left\{\begin{array}{l}
W_{2}^{3}=\varepsilon F\left(A-\left(\nu_{\varepsilon}-\nu_{\varepsilon}^{\prime}\right)|\xi|^{2}\right) W_{2}^{4}, \\
W_{3}^{3}=\varepsilon F\left(B-\left(\nu_{\varepsilon}-\nu_{\varepsilon}^{\prime}\right)|\xi|^{2}\right) W_{3}^{4}, \\
W_{4}^{3}=\varepsilon F\left(\bar{B}-\left(\nu_{\varepsilon}-\nu_{\varepsilon}^{\prime}\right)|\xi|^{2}\right) W_{4}^{4} .
\end{array}\right.
$$

- We have $W_{4}=\overline{W_{3}}$. 


\subsection{Asymptotic expansions of the eigenvalues with respect to the Rossby number $\varepsilon$}

In this paragraph, we provide asymptotic expansions of quantities depending on $\xi \in \mathcal{C}_{r, R}$ with respect to $\varepsilon$. The method is close to [9], except that $r$ and $R$ are powers of the Rossby number and hence, we will need more precise asymptotic expansions in terms of $\varepsilon$. We chose to denote by $E_{2}, E_{2}^{(1)}$, or $E_{2}^{(2)}$ a regular function satisfying $\forall \xi \in \mathcal{C}_{r_{\varepsilon}, R_{\varepsilon}},\left|E_{2}(\xi)\right|,\left|E_{2}^{(1)}(\xi)\right|,\left|E_{2}^{(2)}(\xi)\right| \leq$ $C_{F}\left(\left|\nu_{\varepsilon}-\nu_{\varepsilon}^{\prime}\right| R_{\varepsilon}^{2}\right)$. We recall that the discriminant $D=\frac{q^{2}}{4}+\frac{p^{3}}{27}$. We note

$$
\left\{\begin{array}{l}
p=\frac{p_{1}}{\varepsilon^{2}}+p_{2} \\
q=\frac{q_{1}}{\varepsilon^{2}}+q_{2}
\end{array}\right.
$$

where

$$
\left\{\begin{array}{l}
p_{1}=\frac{|\xi|_{F}^{2}}{F^{2}|\xi|^{2}} \\
p_{2}=-\frac{\left(\nu_{\varepsilon}-\nu_{\varepsilon}^{\prime}\right)^{2}\left|\xi_{h}\right|^{4}}{3} \\
q_{1}=\left(\nu_{\varepsilon}-\nu_{\varepsilon}^{\prime}\right) \frac{\left|\xi_{h}\right|^{2}}{|\xi|^{2}}\left(\frac{|\xi|_{F}^{2}}{3 F^{2}}-\xi_{3}^{2}\right), \\
q_{2}=-\frac{2}{27}\left(\nu_{\varepsilon}-\nu_{\varepsilon}^{\prime}\right)^{3}\left|\xi_{h}\right|^{6}
\end{array}\right.
$$

then we have

$$
\begin{array}{r}
D=\frac{p_{1}^{3}}{27 \varepsilon^{6}}\left[1+C_{1}(\xi)\left|\nu_{\varepsilon}-\nu_{\varepsilon}^{\prime}\right|\left|\xi_{h}\right|^{2} \varepsilon^{2}+C_{2}(\xi)\left|\nu_{\varepsilon}-\nu_{\varepsilon}^{\prime}\right|^{2}\left|\xi_{h}\right|^{4} \varepsilon^{4}\right. \\
\left.+C_{3}(\xi)\left|\nu_{\varepsilon}-\nu_{\varepsilon}^{\prime}\right|^{3}\left|\xi_{h}\right|^{6} \varepsilon^{6}\right],
\end{array}
$$

where $C_{i}(\xi)$ are bounded functions. Thus, there exists a bounded function $E_{2}^{(1)}$ (recall that $\left|\nu_{\varepsilon}-\nu_{\varepsilon}^{\prime}\right| R_{\varepsilon}^{2} \varepsilon \ll 1$ ) such that, $\forall \xi \in \mathcal{C}_{r_{\varepsilon}, R_{\varepsilon}},\left|E_{2}^{(1)}(\xi)\right| \leq$ $C_{F}\left(\left|\nu_{\varepsilon}-\nu_{\varepsilon}^{\prime}\right| R_{\varepsilon}^{2}\right)$, and

$$
D=\frac{p_{1}^{3}}{27 \varepsilon^{6}}\left(1+E_{2}^{(1)} \varepsilon^{2}\right)
$$

Then, writing precisely the majorations of the quantities depending on $\xi$ and taking advantage of the fact that $\xi \in \mathcal{C}_{r_{\varepsilon}, R_{\varepsilon}}$, we finally obtain bounded functions, all of them denoted by $E_{2}^{(2)}$ such that, $\forall \xi \in \mathcal{C}_{r_{\varepsilon}, R_{\varepsilon}},\left|E_{2}^{(2)}(\xi)\right| \leq$ $C_{F}\left(\left|\nu_{\varepsilon}-\nu_{\varepsilon}^{\prime}\right| R_{\varepsilon}^{2}\right)$, and

$$
\left\{\begin{array}{l}
\omega_{1}=\frac{p_{1}^{1 / 2}}{\varepsilon \sqrt{3}}-\frac{q_{1}}{2 p_{1}}+E_{2}^{(2)} \varepsilon \\
\omega_{2}=-\frac{p_{1}^{1 / 2}}{\varepsilon \sqrt{3}}-\frac{q_{1}}{2 p_{1}}+E_{2}^{(2)} \varepsilon,
\end{array}\right.
$$


which allows us to get the following property:

Proposition 3.4 There exist a number $\varepsilon_{0}>0$ and functions, all of them denoted by the same notation $E_{2}(\xi, \varepsilon)$ and satisfying the uniform estimates $\left|E_{2}(\xi)\right| \leq C_{F}\left|\nu_{\varepsilon}-\nu_{\varepsilon}^{\prime}\right| \cdot\left|\xi_{h}\right|^{2}, \forall \xi \in \mathcal{C}_{r_{\varepsilon}, R_{\varepsilon}}$, such that for all $\varepsilon \leq \varepsilon_{0}$ and all $\xi \in \mathcal{C}_{r_{\varepsilon}, R_{\varepsilon}}$ the following asymptotic expansions hold:

$$
\begin{aligned}
& A=\left(\nu_{\varepsilon}-\nu_{\varepsilon}^{\prime}\right) F^{2} \xi_{3}^{2} \frac{\left|\xi_{h}\right|^{2}}{|\xi|_{F}^{2}}+\varepsilon E_{2}, \\
& B=i \frac{|\xi|_{F}}{\varepsilon F|\xi|}+\left(\nu_{\varepsilon}-\nu_{\varepsilon}^{\prime}\right) \frac{\left|\xi_{h}\right|^{4}}{2|\xi|_{F}^{2}}+\varepsilon E_{2}, \\
& \mu=-\left(\nu_{\varepsilon} \xi_{1}^{2}+\nu_{\varepsilon} \xi_{2}^{2}+\nu_{\varepsilon}^{\prime} F^{2} \xi_{3}^{2}\right) \frac{\left|\xi_{h}\right|^{2}}{|\xi|_{F}^{2}}+\varepsilon E_{2}, \\
& \lambda=-\tau(\xi)\left|\xi_{h}\right|^{2}+i \frac{|\xi|_{F}}{\varepsilon F|\xi|}+\varepsilon E_{2},
\end{aligned}
$$

with

$$
\tau(\xi)=\frac{\nu_{\varepsilon}}{2}\left(1+\frac{F^{2} \xi_{3}^{2}}{|\xi|_{F}^{2}}\right)+\frac{\nu_{\varepsilon}^{\prime}}{2}\left(1-\frac{F^{2} \xi_{3}^{2}}{|\xi|_{F}^{2}}\right)
$$

\subsection{Projectors}

We recall that $\mathcal{P}_{i}(\xi, \varepsilon)$ and $\mathbb{P}_{i}(u)=\mathcal{F}^{-1}\left(\mathcal{P}_{i}(\xi, \varepsilon)(\widehat{u}(\xi))\right)$ have been defined in (3.4). In this paragraph we provide estimates of the norms of these operators (defined on Sobolev spaces). We refer to [8] for the fact that $\left(W_{2}, W_{3}, W_{4}\right)$ is a basis of the hyperplane of vectors orthogonal to $\left(\xi_{1}, \xi_{2}, \xi_{3}, 0\right)$, that a vector $g=(X, Y, Z, T)$, orthogonal to $\left(\xi_{1}, \xi_{2}, \xi_{3}, 0\right)$, writes $g=K_{2} W_{2}+K_{3} W_{3}+$ $K_{4} W_{4}$ and that the solution of the system:

$$
\left(\begin{array}{c}
0 \\
K_{2} \\
K_{3} \\
K_{4}
\end{array}\right)=Q^{-1} g
$$

is given by:

$$
\left(\begin{array}{l}
K_{2} \\
K_{3} \\
K_{4}
\end{array}\right)=M\left(\begin{array}{c}
\frac{\xi_{2} X-\xi_{1} Y}{\xi_{3}\left(\xi_{1}^{2}+\xi_{2}^{2}\right)} \\
\frac{\xi_{1} X+\xi_{2} Y}{\varepsilon \xi_{3}\left(\xi_{1}^{2}+\xi_{2}^{2}\right)} \\
\frac{T}{F \varepsilon^{2}|\xi|^{2}}-\frac{\xi_{3}\left(\xi_{2} X-\xi_{1} Y\right)}{\varepsilon^{2}|\xi|^{2}\left(\xi_{1}^{2}+\xi_{2}^{2}\right)}
\end{array}\right),
$$


where the matrix $M$ is defined by:

$$
\left(\begin{array}{ccc}
\frac{|B|^{2}}{|B|^{2}-A B+A^{2}-A \bar{B}} & \frac{-(B+\bar{B})}{|B|^{2}-A B+A^{2}-A \bar{B}} & \frac{1}{|B|^{2}-A B+A^{2}-A \bar{B}} \\
\frac{-A \bar{B}}{-A \bar{B}+A B-B^{2}+|B|^{2}} & \frac{A+\bar{B}}{-A \bar{B}+A B-B^{2}+|B|^{2}} & \frac{-1}{-A \bar{B}+A B-B^{2}+|B|^{2}} \\
\frac{A B}{-A \bar{B}+A B-B^{2}+|B|^{2}} & \frac{-(A+B)}{-A \bar{B}+A B-B^{2}+|B|^{2}} & \frac{1}{-A \bar{B}+A B-B^{2}+|B|^{2}}
\end{array}\right)
$$

Using Remark (3.12), we can compute the coefficients $K_{i}$ the same way as in [8] (taking account of the changes induced by the horizontal laplacian):

$$
\begin{array}{r}
K_{2}=\frac{1}{(A-B)(A-\bar{B}) \varepsilon^{2} F^{2}|\xi|^{2} \xi_{3}\left(\varepsilon^{2}|\xi|^{2} A^{2}+\xi_{3}^{2}\right)}\left(-\xi_{3}^{2}\left(\xi_{1} Y-\xi_{2} X-F \xi_{3} T\right)\right. \\
\left.-\varepsilon A|\xi|^{2}\left(\xi_{1} X+\xi_{2} Y\right)+F \varepsilon^{2}|\xi|^{2} A^{2} \xi_{3} T\right),
\end{array}
$$

and,

$$
\begin{aligned}
& K_{3}=\frac{1}{(A-B)(B-\bar{B}) \varepsilon^{2} F^{2}|\xi|^{2} \xi_{3}\left(\varepsilon^{2}|\xi|^{2} B^{2}+\xi_{3}^{2}\right)}\left(-\xi_{3}^{2}\left(\xi_{2} X-\xi_{1} Y\right)\right. \\
&\left.+\varepsilon B|\xi|^{2}\left(\xi_{1} X+\xi_{2} Y\right)-T \xi_{3} F\left(\xi_{3}^{2}+\varepsilon^{2}|\xi|^{2} B^{2}\right)\right) .
\end{aligned}
$$

We recall that, thanks to the coefficient-root relations of the polynomial

$$
(X-A)(X-B)(X-\bar{B}),
$$

we have the following equality:

$$
(A-B)(A-\bar{B}) \varepsilon^{2} F^{2}|\xi|^{2}=|\xi|_{F}^{2}-2\left(\nu-\nu^{\prime}\right)|\xi|_{h}^{2} A \varepsilon^{2} F^{2}|\xi|^{2}+3 A^{2} \varepsilon^{2} F^{2}|\xi|^{2},
$$

So, if $\varepsilon$ is small enough, the previous asymptotic expansions imply that:

$$
\left.\left.\left|(A-B)(A-\bar{B}) \varepsilon^{2} F^{2}\right| \xi\right|^{2}\left|\geq \frac{1}{2}\right| \xi\right|_{F} ^{2} .
$$

Moreover, a simple computation leads to:

$$
\left|W_{2}\right|^{2}=\left(\xi_{3}^{2}+\varepsilon^{2} A^{2}|\xi|^{2}\right)\left(|\xi|_{F}^{2}+\varepsilon^{2} F^{2} A^{2}|\xi|^{2}\right),
$$

and to the following estimate:

$$
\begin{aligned}
\left|K_{2} W_{2}\right|_{\mathbb{C}^{4}} \leq \frac{1}{\left.\left|(A-B)(A-\bar{B}) \varepsilon^{2} F^{2}\right| \xi\right|^{2} \mid}\left(\frac{|\xi|_{F}^{2}+\varepsilon^{2} F^{2} A^{2}|\xi|^{2}}{\xi_{3}^{2}+\varepsilon^{2} A^{2}|\xi|^{2}}\right)^{\frac{1}{2}} \\
\left(\left|\xi_{3}\right| \cdot\left|\xi_{1} Y-\xi_{2} X-F \xi_{3} T\right|+|A| \varepsilon\left(\left|\xi_{1}\right|+\left|\xi_{2}\right|\right) \frac{|\xi|^{2}}{\left|\xi_{3}\right|}|f|+F \varepsilon^{2}|\xi|^{2} A^{2}|f|\right) .
\end{aligned}
$$


We consider two cases:

- If $\Omega(f) \neq 0$, that is $\xi_{1} Y-\xi_{2} X-F \xi_{3} T \neq 0$, then when $\varepsilon$ is small, we cannot get a better estimate than:

$$
\left|K_{2} W_{2}\right|_{\mathbb{C}^{4}} \leq \frac{2}{|\xi|_{F}^{2}} \frac{|\xi|_{F}}{\left|\xi_{3}\right|}\left(\left|\xi_{3}\right| \cdot|\xi|+\varepsilon|A| \frac{|\xi|^{3}}{\left|\xi_{3}\right|}+F \varepsilon^{2} A^{2}|\xi|^{2}\right)|f| \leq C_{F} \frac{|\xi|}{|\xi|_{F}}|f| .
$$

- If $\Omega(f)=0$, then we obtain that:

$$
\left|K_{2} W_{2}\right|_{\mathbb{C}^{4}} \leq \frac{2}{|\xi|_{F}^{2}} \frac{|\xi|_{F}}{\left|\xi_{3}\right|} \varepsilon|A| \frac{|\xi|^{3}}{\left|\xi_{3}\right|}|f|
$$

then, using the asymptotic expansions we get that the term is much smaller than in the first case:

$$
\left|K_{2} W_{2}\right|_{\mathbb{C}^{4}} \leq C_{F}\left|\nu-\nu^{\prime}\right| \varepsilon R_{\varepsilon}^{2}|f|
$$

An adaptation of the previous computations (except that we have to be careful for the asymptotic expansions of $B$, and that we do not have in this case any simplification if $\Omega(f)=0$ ) leads to the estimates of $K_{i} W_{i}$ for $i=3,4$. We gather these estimates into the following lemma:

Lemma 3.5 There exists a constant $C_{F}$ and $\varepsilon_{0}>0$ such that for all $g(\xi)=$ $(X(\xi), Y(\xi), Z(\xi), T(\xi))$ orthogonal to $\left(\xi_{1}, \xi_{2}, \xi_{3}, 0\right)$ and $\varepsilon \leq \varepsilon_{0}$, for all $\xi \in$ $\mathcal{C}_{r_{\varepsilon}, R_{\varepsilon}}$ we have the estimates:

$$
\left|K_{2} W_{2}\right|_{\mathbb{C}^{4}} \leq \begin{cases}C_{F} \frac{|\xi|}{\left|\xi_{F}\right|}|g(\xi)| & \text { if } \quad \xi_{1} Y-\xi_{2} X-F \xi_{3} T \neq 0, \\ C_{F}\left|\nu_{\varepsilon}-\nu_{\varepsilon}^{\prime}\right| \varepsilon \frac{|\xi|^{3}}{|\xi|_{F}^{3}}\left|\xi_{h}\right|^{2}|g(\xi)| & \text { if } \quad \xi_{1} Y-\xi_{2} X-F \xi_{3} T \equiv 0 .\end{cases}
$$

And for $i=3,4$,

$$
\left|K_{i} W_{i}\right| \leq C_{F}\left(\frac{\left|\xi_{3}\right|}{|\xi|}+\frac{|\xi|}{\left|\xi_{3}\right|}+\frac{\left|\xi_{h}\right|}{|\xi|}\right)|g(\xi)| .
$$

As $r_{\varepsilon}=R_{\varepsilon}^{-\gamma}$, it follows that:

Corollary 3.6 If $f$ is a divergence-free vector field, we have the following estimates on the norms of the projectors: for all $\xi \in \mathcal{C}_{r_{\varepsilon}, R_{\varepsilon}}$

$$
\left|\widehat{\mathbb{P}_{2} f}(\xi)\right| \leq \begin{cases}C_{F}|\widehat{f}(\xi)| & \text { if } \Omega(f) \neq 0, \\ C_{F}\left|\nu_{\varepsilon}-\nu_{\varepsilon}^{\prime}\right| \varepsilon R_{\varepsilon}^{2}|\widehat{f}(\xi)| & \text { if } \quad \Omega(g)=0 .\end{cases}
$$

And for $i=3,4$, we have

$$
\left|\widehat{\mathbb{P}_{i} f}(\xi)\right| \leq C_{F} R_{\varepsilon}^{1+\gamma}|\widehat{f}(\xi)| .
$$

The conclusion of the proof of proposition 3.1 is then easy. 


\section{Dispersion and Strichartz estimates}

The aim of this section is to prove the following Strichartz estimates:

Lemma 4.1 Assume that $f$ satisfies the following system:

$$
\left\{\begin{array}{l}
\partial_{t} f-L f+\frac{1}{\varepsilon} \mathbb{P} \mathcal{A} f=0 \\
f_{\mid t=0}=f_{0},
\end{array}\right.
$$

where div $f_{0}=0$, and $L$ is the operator defined in the introduction (with $\nu_{\varepsilon}=\varepsilon^{\alpha}$ and $\left.\nu_{\varepsilon}^{\prime}=\rho \varepsilon^{\alpha}\right)$.

Assume also that the frequencies of $f_{0}$ are localized in $\mathcal{C}_{r_{\varepsilon}, R_{\varepsilon}}$. Then, there exists a constant $C_{F, \rho}>0$ such that for $i=3,4$ :

$$
\left\|\mathbb{P}_{i} f\right\|_{L^{1}\left(\mathbb{R}_{+}, L_{h, v}^{\infty, 2}\right)} \leq C_{F, \rho} \varepsilon^{\frac{1-3 \alpha}{4}} R_{\varepsilon}^{\frac{7+9 \gamma}{2}}\left\|f_{0}\right\|_{L^{2}} .
$$

\subsection{Duality}

We have seen in Section 3.3 that matrix $\mathbb{B}$ has four distinct eigenvalues: two of them are real (only $\mu$ will be useful in the following), and the other two are conjugated complex numbers with the following asymptotic expansion:

$$
\lambda=-\tau(\xi)\left|\xi_{h}\right|^{2}+i \frac{|\xi|_{F}}{\varepsilon F|\xi|}+\varepsilon E_{2},
$$

with $\left|E_{2}\right| \leq C_{F}\left|\nu_{\varepsilon}-\nu_{\varepsilon}^{\prime}\right| \cdot\left|\xi_{h}\right|^{2}$ and $\tau(\xi) \geq \min \left(\nu_{\varepsilon}, \nu_{\varepsilon}^{\prime}\right)$.

For $i \in\{3,4\}, \mathbb{P}_{i} f$ satisfies system (4.1) with $\mathbb{P}_{i} f_{0}$ as its initial data, so in the Fourier variable, we have:

$$
\widehat{\mathbb{P}_{i} f(t, \xi)}=e^{-t \tau(\xi)\left|\xi_{h}\right|^{2}+i \frac{t|\xi|_{F}}{\varepsilon F|\xi|}+\varepsilon t E_{2}} \widehat{\mathbb{P}_{i} f_{0}(\xi)} .
$$

In order to compute the desired norm, we introduce the set:

$$
\mathcal{B}=\left\{\psi \in \mathcal{D}\left(\mathbb{R}_{+} \times \mathbb{R}^{3}\right) / \quad\|\psi\|_{L_{T}^{\infty}\left(L_{h, v}^{1,2}\right)} \leq 1\right\},
$$

and duality arguments allow us to write that:

$$
\left\|\mathbb{P}_{i} f\right\|_{L_{T}^{1}\left(L_{h, v}^{\infty, 2}\right)}=\sup _{\psi \in \mathcal{B}} \int_{0}^{\infty} \int_{\mathbb{R}^{3}} \mathbb{P}_{i} f(t, x) \psi(t, x) d x d t .
$$

Thanks to the properties of the truncation function and the initial data $f_{0}$, we have $f_{0}=\mathcal{P}_{\frac{r_{\varepsilon}}{2}, 2 R_{\varepsilon}} f_{0}$, so using the Plancherel formula we obtain:

$$
\begin{gathered}
\left\|\mathbb{P}_{i} f\right\|_{L_{T}^{1}\left(L_{h, v}^{\infty, 2}\right)}=\sup _{\psi \in \mathcal{B}} \int_{0}^{\infty} \int_{\mathbb{R}^{3}} \chi \\
e^{-t \tau(\xi)\left|\xi_{h}\right|^{2}+i \frac{t|\xi|}{\varepsilon F|\xi|}+\varepsilon t E_{2}} \widehat{\mathbb{P}_{i} f_{0}}(\xi) \widehat{\psi}(t, \xi) d \xi d t
\end{gathered}
$$


So, if we note $\phi_{\varepsilon}=\chi\left(\frac{|\xi|}{2 R_{\varepsilon}}\right)\left(1-\chi\left(\frac{2\left|\xi_{3}\right|}{r_{\varepsilon}}\right)\right)\left(1-\chi\left(\frac{2\left|\xi_{h}\right|}{r_{\varepsilon}}\right)\right)$ and $d(\xi)=\frac{|\xi|_{F}}{F|\xi|}$, using the Fubini theorem and the Hölder estimate implies that

$$
\begin{aligned}
\left\|\mathbb{P}_{i} f\right\|_{L_{T}^{1}\left(L_{h, v}^{\infty, 2}\right)} \leq C \sup _{\psi \in \mathcal{B}} & \left\|\mathbb{P}_{i} f_{0}\right\|_{L^{2}}\left(\int_{\mathbb{R}^{3}} \int_{0}^{\infty} \int_{0}^{\infty} \phi_{\varepsilon}(\xi)^{2}\right. \\
& \left.e^{-(t+s) \tau(\xi)\left|\xi_{h}\right|^{2}+i \frac{t-s}{\varepsilon} d(\xi)+\varepsilon t E_{2}-\varepsilon s \overline{E_{2}}} \widehat{\psi}(t, \xi) \overline{\widehat{\psi}(s, \xi)} d s d t d \xi\right)^{\frac{1}{2}} .
\end{aligned}
$$

Another use of Fubini and the Plancherel formula gives:

$$
\begin{aligned}
& \left\|\mathbb{P}_{i} f\right\|_{L_{T}^{1}\left(L_{h, v}^{\infty, 2}\right)} \leq C \sup _{\psi \in \mathcal{B}}\left\|\mathbb{P}_{i} f_{0}\right\|_{L^{2}}\left(\int _ { 0 } ^ { \infty } \int _ { 0 } ^ { \infty } \int _ { \mathbb { R } ^ { 3 } } \left(\int_{\mathbb{R}^{3}} e^{i x \cdot \xi} \phi_{\varepsilon}(\xi)^{2}\right.\right. \\
& \left.\left.e^{-(t+s) \tau(\xi)\left|\xi_{h}\right|^{2}+i \frac{t-s}{\varepsilon} d(\xi)+\varepsilon t E_{2}-\varepsilon s \overline{E_{2}}} \widehat{\psi}(t, \xi) d \xi\right) \bar{\psi}(s, x) d x d s d t\right)^{\frac{1}{2}} .
\end{aligned}
$$

Then, we obtain that:

$$
\begin{aligned}
\left\|\mathbb{P}_{i} f\right\|_{L_{T}^{1}\left(L_{h, v}^{\infty, 2}\right)} & \leq C \sup _{\psi \in \mathcal{B}}\left\|\mathbb{P}_{i} f_{0}\right\|_{L^{2}} \\
& \left(\int_{0}^{\infty} \int_{0}^{\infty}\left\|K\left(t+s, \frac{t-s}{\varepsilon}, .\right)\right\|_{L_{h, v}^{\infty, 2}}\|\bar{\psi}(s, .)\|_{L_{h, v}^{1,2}} d t d s\right)^{\frac{1}{2}}
\end{aligned}
$$

where

$$
\begin{aligned}
& K\left(t+s, \frac{t-s}{\varepsilon},\right.x) \stackrel{\text { def }}{=} \int_{\mathbb{R}^{3}} e^{i x \cdot \xi-(t+s) \tau(\xi)\left|\xi_{h}\right|^{2}+i \frac{t-s}{\varepsilon} d(\xi)+\varepsilon t E_{2}-\varepsilon s \overline{E_{2}}} \\
& \chi\left(\frac{|\xi|}{2 R_{\varepsilon}}\right)^{2}\left(1-\chi\left(\frac{2\left|\xi_{3}\right|}{r_{\varepsilon}}\right)\right)^{2}\left(1-\chi\left(\frac{2\left|\xi_{h}\right|}{r_{\varepsilon}}\right)\right)^{2} \widehat{\psi}(t, \xi) d \xi,
\end{aligned}
$$

and where $\chi$ is defined as in (3.1).

If we manage to estimate $\left\|K\left(t+s, \frac{t-s}{\varepsilon}, .\right)\right\|_{L_{h, v}^{\infty, 2}}$ then we immediately get the estimate of $\left\|\mathbb{P}_{i} f\right\|_{L_{T}^{1}\left(L_{h, v}^{\infty, 2}\right)}$. In the following paragraph we will give the dispersive estimates related to $K$, that is $\left\|K\left(t+s, \frac{t-s}{\varepsilon}, .\right)\right\|_{L_{h, v}^{\infty, 2}}$.

\subsection{Dispersion}

In this paragraph, we prove the following dispersive estimate

Lemma 4.2 There exists a constant $C_{F, \rho}>0$ such that for all $t \neq s \in \mathbb{R}^{3}$,

$$
\left\|K\left(t+s, \frac{t-s}{\varepsilon}, .\right)\right\|_{L_{x_{h}, x_{3}}^{\infty, 2}} \leq C_{F, \rho} \frac{\varepsilon^{\frac{1}{2}}}{|t-s|^{\frac{1}{2}}} \frac{R_{\varepsilon}^{5}}{r_{\varepsilon}^{4}} e^{-(t+s) \nu_{\varepsilon, 0} \frac{r_{\varepsilon}^{2}}{4}}\|\psi(t)\|_{L_{x_{h}, x_{3}}^{1,2}},
$$

where $\nu_{\varepsilon, 0}=\min \left(\nu_{\varepsilon}, \nu_{\varepsilon}^{\prime}\right)=\min (1, \rho) \varepsilon^{\alpha}>0$. 
The methods used here are the same as in [15] and [35]. First we introduce:

$$
\begin{aligned}
I\left(t+s, \frac{t-s}{\varepsilon}, z_{h}, \xi_{3}\right) \stackrel{\text { def }}{=} & \left(1-\chi\left(\frac{2\left|\xi_{3}\right|}{r_{\varepsilon}}\right)\right)^{2} \int_{\mathbb{R}_{\xi_{h}}^{2}} e^{i z_{h} \cdot \xi_{h}-(t+s) \tau(\xi)\left|\xi_{h}\right|^{2}+i \frac{t-s}{\varepsilon} d(\xi)} \\
& e^{\varepsilon t E_{2}-\varepsilon s \overline{E_{2}}} \chi\left(\frac{|\xi|}{2 R_{\varepsilon}}\right)^{2}\left(1-\chi\left(\frac{2\left|\xi_{h}\right|}{r_{\varepsilon}}\right)\right)^{2} d \xi_{h} .
\end{aligned}
$$

As $\mathcal{F} K=\mathcal{F}_{h} I\left(t, \xi_{h}, \xi_{3}\right) . \mathcal{F} \psi(t, \xi)$, we have $\mathcal{F}_{3} K=I\left(t, ., \xi_{3}\right) *_{h} \mathcal{F}_{3} \psi\left(t, ., \xi_{3}\right)$. Using convolution properties it is then easy to see that:

$$
\left\|K\left(t+s, \frac{t-s}{\varepsilon}, .\right)\right\|_{L_{x_{h}, x_{3}}^{\infty, 2}} \leq C\left\|I\left(t+s, \frac{t-s}{\varepsilon}, .\right)\right\|_{L_{z_{h}, \xi_{3}}^{\infty, \infty}}\|\psi(t)\|_{L_{x_{h}, x_{3}}^{1,2}} .
$$

So Lemma 4.2 is in fact a corollary of the following result:

Lemma 4.3 There exists a constant $C_{F, \rho}>0$ such that for all $t \neq s \in \mathbb{R}^{3}$,

$$
\left\|I\left(t+s, \frac{t-s}{\varepsilon}, .\right)\right\|_{L_{z_{h}, \xi_{3}}^{\infty, \infty}} \leq C_{F, \rho} \frac{\varepsilon^{\frac{1}{2}}}{|t-s|^{\frac{1}{2}}} \frac{R_{\varepsilon}^{5}}{r_{\varepsilon}^{4}} e^{-\frac{1}{4}(t+s) \nu_{\varepsilon, 0} r_{\varepsilon}^{2}} .
$$

Proof. We will only give a sketch of the proof, since it is very close to the ones of [15] and [35] for example. The difference is that, like in [8] and [9], we have to be careful with the asymptotic expansions in order not to get a negative power of $\varepsilon$. Let us introduce the function

$$
\delta(\xi)=-\partial_{\xi_{2}} \frac{|\xi|_{F}}{F|\xi|}=-\frac{F^{2}\left(F^{2}-1\right) \xi_{2} \xi_{3}^{2}}{|\xi|_{F}|\xi|^{3}},
$$

and the operator:

$$
\mathcal{L}=\frac{1}{1+\frac{t-s}{\varepsilon} \delta^{2}}\left(1+i \delta \partial_{\xi_{2}}\right)
$$

We can assume $t>s$. If not, just take instead :

$$
\frac{1}{1+\frac{s-t}{\varepsilon} \delta^{2}}\left(1+i \delta \partial_{\xi_{2}}\right)
$$

The invariance under the rotation around the z-axis allows us to assume that $z_{2}=0$. So

$$
\mathcal{L}\left(e^{i z_{h} \cdot \xi_{h}+i \frac{t-s}{\varepsilon} d(\xi)}\right)=e^{i z_{h} \cdot \xi_{h}+i \frac{t-s}{\varepsilon} d(\xi)} .
$$

We have

$$
\begin{aligned}
& I\left(t+s, \frac{t-s}{\varepsilon}, z_{h}, \xi_{3}\right)=\int_{\mathbb{R}_{\xi_{h}}^{2}}\left[1-\chi\left(\frac{2\left|\xi_{3}\right|}{r_{\varepsilon}}\right)\right]^{2} e^{i z_{h} \cdot \xi_{h}+i \frac{t-s}{\varepsilon}} d(\xi) \\
& { }^{T} \mathcal{L}\left(e^{-(t+s) \tau(\xi)\left|\xi_{h}\right|^{2}+\varepsilon t E_{2}-\varepsilon t \overline{E_{2}}} \chi\left(\frac{|\xi|}{2 R_{\varepsilon}}\right)^{2}\left[1-\chi\left(\frac{2\left|\xi_{h}\right|}{r_{\varepsilon}}\right)\right]^{2}\right) d \xi_{h}
\end{aligned}
$$


and where the transposed operator of $\mathcal{L}$ writes:

$$
{ }^{T} \mathcal{L}(g)=\left(\frac{1}{1+\frac{t-s}{\varepsilon} \delta^{2}}-i \partial_{\xi_{2}} \delta \frac{1-\frac{t-s}{\varepsilon} \delta^{2}}{\left(1+\frac{t-s}{\varepsilon} \delta^{2}\right)^{2}}\right) g-\frac{i \delta}{1+\frac{t-s}{\varepsilon} \delta^{2}} \partial_{\xi_{2}} g .
$$

If $\varepsilon$ is small enough, we have

$$
\mathcal{R} e\left((t+s) \tau(\xi)-t \varepsilon \frac{E_{2}}{\left|\xi_{h}\right|^{2}}+s \varepsilon \frac{\overline{E_{2}}}{\left|\xi_{h}\right|^{2}}\right) \geq(t+s) \frac{\nu_{\varepsilon, 0}}{2},
$$

where $\nu_{\varepsilon, 0}=\min \left(\nu_{\varepsilon}, \nu_{\varepsilon}^{\prime}\right)=\min (1, \rho) \varepsilon^{\alpha}$. Then, using the expression of $\delta$, the fact that $\xi \in \mathcal{C}_{r_{\varepsilon}, R_{\varepsilon}}$ and the inequality $u e^{-m u} \leq \frac{2}{e^{m}} e^{\frac{-m u}{2}}$, for $u, m>0$, we obtain:

$$
\begin{array}{r}
\left|{ }^{T} \mathcal{L}\left(e^{-(t+s) \tau(\xi)\left|\xi_{h}\right|^{2}+\varepsilon t E_{2}-\varepsilon t \overline{E_{2}}} \chi\left(\frac{|\xi|}{2 R_{\varepsilon}}\right)^{2}\left[1-\chi\left(\frac{2\left|\xi_{h}\right|}{r_{\varepsilon}}\right)\right]^{2}\right)\right| \leq \\
\leq C_{F ; \rho} e^{-\frac{\nu_{\varepsilon, 0}}{2}\left|\xi_{h}\right|^{2}(t+s)}\left(1+\frac{1}{r_{\varepsilon}^{2}}+(t+s)\left(\nu_{\varepsilon}+\nu_{\varepsilon}^{\prime}\right)\left|\xi_{h}\right|\right) \frac{1}{1+\frac{t-s}{\varepsilon} \delta^{2}}
\end{array}
$$

Thus, we have

$$
\left|I\left(t+s, \frac{t-s}{\varepsilon}, z_{h}, \xi_{3}\right)\right| \leq \frac{C_{F, \rho}}{r_{\varepsilon}^{2}} e^{-\frac{\nu_{\varepsilon, 0}}{4}\left|\xi_{h}\right|^{2}(t+s)} \int_{r_{\varepsilon} \leq\left|\xi_{h}\right| \leq R_{\varepsilon}} \frac{d \xi_{h}}{1+\frac{t-s}{\varepsilon} C_{F} \frac{\xi_{2}^{2} \xi_{3}^{4}}{|\xi|_{F}^{2}|\xi|^{6}}}
$$

Performing a change of variable $\zeta=\frac{(t-s)^{\frac{1}{2}} \xi_{2}}{\varepsilon^{\frac{1}{2}} r_{\varepsilon}^{2}}$, we get the proof of Lemma 4.3, and then we can conclude the proof of Lemma 4.2.

\subsection{Proof of the Strichartz estimates}

Going back to (4.2) and using the dispersive estimates, we obtain:

$$
\begin{aligned}
& \left\|\mathbb{P}_{i} f\right\|_{L_{T}^{1}\left(L_{h, v}^{\infty, 2}\right)} \leq C_{F, \rho} \sup _{\psi \in \mathcal{B}}\left\|\mathbb{P}_{i} f_{0}\right\|_{L^{2}} \\
& \left(\int_{0}^{\infty} \int_{0}^{\infty} \frac{\varepsilon^{\frac{1}{2}}}{|t-s|^{\frac{1}{2}}} \frac{R_{\varepsilon}^{5}}{r_{\varepsilon}^{4}} e^{-(t+s) \nu_{\varepsilon, 0} \frac{r_{\varepsilon}^{2}}{4}}\|\psi(t)\|_{L_{x_{h}, x_{3}}^{1,2}}\|\bar{\psi}(s)\|_{L_{x_{h}, x_{3}}^{1,2}} d t d s\right)^{\frac{1}{2}} .
\end{aligned}
$$

Then the Hardy-Littlewood theorem implies that:

$$
\left\|\mathbb{P}_{i} f\right\|_{L_{T}^{1}\left(L_{h, v}^{\infty, 2}\right)} \leq C_{F, \rho}\left\|\mathbb{P}_{i} f_{0}\right\|_{L^{2}} \frac{\varepsilon^{\frac{1}{4}}}{\nu_{\varepsilon, 0}^{\frac{3}{4}}} \frac{R_{\varepsilon}^{\frac{5}{2}}}{r_{\varepsilon}^{\frac{7}{2}}}
$$

We use Proposition 3.1 and it concludes the proof of Lemma 4.1.

Remark 4.4 The result given in Lemma 4.1 only concerns $\mathbb{P}_{3} f$ and $\mathbb{P}_{4} f$. It is important to remember that $\hat{f}$ has a component along $W_{2}$. 


\section{Global existence, proof of the theorem}

The aim of this section is to prove the following theorem, which is more precise than Theorem 1.4 as it gives asymptotics. Recall that for $s>\frac{1}{2}$ and $\eta>0$,

$$
Y_{s, \eta}=H^{\eta, s+\eta} \cap L_{h}^{2} \dot{H}_{v}^{-2 \eta} \cap \dot{H}_{h}^{-\eta} H_{v}^{s},
$$

where $\dot{H}^{-\eta}$ are usual homogeneous Sobolev spaces. Let us consider the spaces

$$
E^{s}=\left\{u \in \mathcal{S}^{\prime}\left(\mathbb{R}_{+} \times \mathbb{R}^{3}\right): \quad\|u\|_{L^{\infty}\left(\mathbb{R}_{+}, H^{0, s}\right)}^{2}+\int_{0}^{\infty}\left\|\nabla_{h} u(t)\right\|_{H^{0, s}}^{2} d t<\infty\right\} .
$$

The main theorem is as follows:

Theorem 5.1 (Global Existence) Let $s>\frac{1}{2}$, and $\eta>0$. There exists $\alpha_{0}>0$ such that for all $0<\alpha<\alpha_{0}$, for all $r_{0}>0$, for all $0<\varepsilon<\varepsilon_{0}$ where $\varepsilon_{0}$ depends on $r_{0}$ and for all $U_{0} \in Y_{s, \eta}$ with a zero quasi-geostrophic part and with $\left\|U_{0}\right\|_{Y_{s, \eta}} \leq r_{0}$, system $\left(A P E_{\varepsilon}\right)$ has a unique global solution $U_{\varepsilon}$ in $\mathbf{C}_{b}\left(\mathbb{R}_{+}, H^{0, s}\right)$ with $\nabla_{h} U_{\varepsilon} \in L^{2}\left(\mathbb{R}_{+}, H^{0, s}\right)$.

Moreover, if $\overline{U_{0, \varepsilon}}=\mathcal{P}_{r, R} U_{0}$, where the cut-off operator $\mathcal{P}_{r, R}$ is defined as in (3.2), and $\overline{U_{\varepsilon}}$ is the solution of the following linear system:

$$
\left\{\begin{array}{l}
\partial_{t} \overline{U_{\varepsilon}}-L \overline{U_{\varepsilon}}+\frac{1}{\varepsilon} \mathcal{A} \overline{U_{\varepsilon}}=\left(-\nabla \overline{\Phi_{\varepsilon}}, 0\right) \\
\operatorname{div} \overline{v_{\varepsilon}}=0 \\
\overline{U_{\varepsilon}} \mid t=0=\mathbb{P}_{3+4} \overline{U_{0, \varepsilon}}
\end{array}\right.
$$

with $R=R_{\varepsilon}=\varepsilon^{-\beta}$ and $r=r_{\varepsilon}=R_{\varepsilon}^{-\gamma}$, then there exist $\beta$ and $\gamma$ such that $V_{\varepsilon}=U_{\varepsilon}-\overline{U_{\varepsilon}}$ goes to zero in $E^{s}$, when $\varepsilon$ goes to zero.

\subsection{The different systems}

As announced in the introduction, we will consider oscillating initial data (their quasi-geostrophic part is 0 ). So in system $\left(A P E_{\varepsilon}\right)$, we have $U_{0, Q G}=0$.

As in [35] we decompose the initial data with frequency truncations: $U_{0}=\overline{U_{0, \varepsilon}}+V_{0, \varepsilon}$, where:

$$
\left\{\begin{array}{l}
\overline{U_{0, \varepsilon}}=\mathcal{P}_{r_{\varepsilon}, R_{\varepsilon}} U_{0} \\
V_{0, \varepsilon}=U_{0}-\overline{U_{0, \varepsilon}} .
\end{array}\right.
$$

Then we define $\overline{U_{\varepsilon}}$ as the solution of the following linear system (projecting this system with the Leray projector $\mathbb{P}$ gives exactly system (4.1)):

$$
\left\{\begin{array}{l}
\partial_{t} \overline{U_{\varepsilon}}-L \overline{U_{\varepsilon}}+\frac{1}{\varepsilon} \mathcal{A} \overline{U_{\varepsilon}}=\left(-\nabla \overline{\Phi_{\varepsilon}}, 0\right) \\
\operatorname{div} \overline{v_{\varepsilon}}=0 \\
\overline{U_{\varepsilon} \mid t=0}=\mathbb{P}_{3+4} \overline{U_{0, \varepsilon}}
\end{array} .\right.
$$


And if we define $V_{\varepsilon}=U_{\varepsilon}-\overline{U_{\varepsilon}}$, then $V_{\varepsilon}$ satisfies the following system: (5.3)

$\left\{\begin{array}{l}\partial_{t} V_{\varepsilon}-L V_{\varepsilon}+\frac{1}{\varepsilon} \mathcal{A} V_{\varepsilon}=-\frac{1}{\varepsilon}\left(\nabla \phi_{\varepsilon}, 0\right)-V_{\varepsilon} \cdot \nabla V_{\varepsilon}-V_{\varepsilon} \cdot \nabla \overline{U_{\varepsilon}}-\overline{U_{\varepsilon}} \cdot \nabla V_{\varepsilon}-\overline{U_{\varepsilon}} \cdot \nabla \overline{U_{\varepsilon}} \\ V_{\varepsilon \mid t=0}=V_{0, \varepsilon}+\mathbb{P}_{2} \overline{U_{0, \varepsilon}}\end{array}\right.$

As both $U_{\varepsilon}$ and $\overline{U_{\varepsilon}}$ are divergence free, so is $V_{\varepsilon}$ and then $\mathbb{P} V_{\varepsilon}=V_{\varepsilon}$, so finally $V_{\varepsilon}$ satisfies the following system:

$$
\left\{\begin{array}{l}
\partial_{t} V_{\varepsilon}-L V_{\varepsilon}+\frac{1}{\varepsilon} \mathbb{P} \mathcal{A} V_{\varepsilon}=-\mathbb{P}\left(V_{\varepsilon} \cdot \nabla V_{\varepsilon}+V_{\varepsilon} \cdot \nabla \overline{U_{\varepsilon}}+\overline{U_{\varepsilon}} \cdot \nabla V_{\varepsilon}+\overline{U_{\varepsilon}} \cdot \nabla \overline{U_{\varepsilon}}\right) \\
V_{\varepsilon \mid t=0}=V_{0, \varepsilon}+\mathbb{P}_{2} \overline{U_{0, \varepsilon}}
\end{array}\right.
$$

Remark 5.2 Obviously we have $\Omega\left(\overline{U_{0, \varepsilon}}\right)=0$, so we will be able to use optimized estimates from Proposition 3.1 for $\mathbb{P}_{2} \overline{U_{0, \varepsilon}}$.

As $\overline{U_{\varepsilon} \mid t=0}=\mathbb{P}_{3+4} \overline{U_{0, \varepsilon}}$ has no " $\mathbb{P}_{2}$-component", and as the system is diagonalizable, then for all $t$, so is $\overline{U_{\varepsilon}}(t)$ and $\overline{U_{\varepsilon}}=\mathbb{P}_{3+4} \overline{U_{\varepsilon}}$. Using Lemma 4.1 and the fact that $\mathbb{P}_{3+4} \overline{U_{0, \varepsilon}}=\left(I_{d}-\mathbb{P}_{2}\right) \overline{U_{0, \varepsilon}}$ (using Proposition 3.1 easily implies $\left\|\overline{U_{\varepsilon}}(0)\right\|_{L^{2}} \leq 2\left\|\overline{U_{0, \varepsilon}}\right\|_{L^{2}} \leq 2\left\|U_{0}\right\|_{L^{2}}$ if $\varepsilon$ is small enough) we have:

$$
\left\|\overline{U_{\varepsilon}}\right\|_{L^{1}\left(\mathbb{R}_{+}, L_{h, v}^{\infty, 2}\right)} \leq C_{F, \rho} \varepsilon^{\frac{1-3 \alpha}{4}} R_{\varepsilon}^{\frac{7+9 \gamma}{2}}\left\|U_{0}\right\|_{L^{2}} .
$$

Using the Bernstein Lemma, Proposition 3.1, and the energy estimates we get:

$$
\begin{aligned}
\left\|\overline{U_{\varepsilon}}\right\|_{L^{\infty}\left(\mathbb{R}_{+}, L_{h, v}^{\infty, 2}\right)} & \leq C R_{\varepsilon}^{2\left(\frac{1}{2}-\frac{1}{\infty}\right)}\left\|\overline{U_{\varepsilon}}\right\|_{L^{\infty}\left(\mathbb{R}_{+}, L^{2}\right)} \\
& \leq C R_{\varepsilon}\left\|\mathbb{P}_{3+4} \overline{U_{0, \varepsilon}}\right\|_{L^{2}} \leq C_{F} R_{\varepsilon}^{2+\gamma}\left\|U_{0}\right\|_{L^{2}} .
\end{aligned}
$$

Then, using interpolation we obtain the formulation of the Strichartz estimates we will use in this article:

Lemma 5.3 With the same notations as above, the solution of (5.2) satisfies the following Strichartz estimates:

$$
\left\|\overline{U_{\varepsilon}}\right\|_{L^{p}\left(\mathbb{R}_{+}, L_{h, v}^{\infty, 2}\right)} \leq C_{F, \rho} \varepsilon^{\frac{1-3 \alpha}{4 p}} R_{\varepsilon}^{\frac{3+7 \gamma}{2 p}+2+\gamma}\left\|U_{0}\right\|_{L^{2}},
$$

and, localizing in frequency, we get that for all $q$,

$$
\left\|\Delta_{q}^{v} \overline{U_{\varepsilon}}\right\|_{L^{p}\left(\mathbb{R}_{+}, L_{h, v}^{\infty, 2}\right)} \leq C_{F, \rho} \varepsilon^{\frac{1-3 \alpha}{4 p}} R_{\varepsilon}^{\frac{3+7 \gamma}{2 p}+2+\gamma}\left\|\Delta_{q}^{v} U_{0}\right\|_{L^{2}}
$$

so, there exists a square summable sequence $\left(c_{q}\right)_{q \in\{-1\} \cup \mathbb{N}}$ such that $\sum_{q} c_{q}^{2} \leq 1$ and for all $q$,

$$
\left\|\Delta_{q}^{v} \overline{U_{\varepsilon}}\right\|_{L^{p}\left(\mathbb{R}_{+}, L_{h, v}^{\infty, 2}\right)} \leq c_{q} 2^{-q s} C_{F, \rho} \varepsilon^{\frac{1-3 \alpha}{4 p}} R_{\varepsilon}^{\frac{3+7 \gamma}{2 p}+2+\gamma}\left\|U_{0}\right\|_{H^{0, s}}
$$




\subsection{Truncation of the data}

In this paragraph, we study the truncation in frequency of the initial data $U_{0}$ introduced in the previous section: $V_{\varepsilon \mid t=0}=V_{0, \varepsilon}+\mathbb{P}_{2} \overline{U_{0, \varepsilon}}$. Recall that $U_{0}$ has no quasigeostropic part so using Proposition 3.1, we get that the " $\mathbb{P}_{2}$ component" of the medium-frequency part of $U_{0}$ (with $0<r_{\varepsilon} \leq\left|\xi_{h}\right|,\left|\xi_{3}\right|,|\xi| \leq R_{\varepsilon}$ ) goes to zero when $\varepsilon$ goes to zero:

$$
\left\|\mathbb{P}_{2} \overline{U_{0, \varepsilon}}\right\|_{H^{0, s}} \leq C_{F, \rho} \varepsilon^{1+\alpha} R_{\varepsilon}^{2}\left\|U_{0}\right\|_{H^{0, s}} .
$$

We prove now that with $r_{\varepsilon}=R_{\varepsilon}^{-\gamma}$ with $\gamma>0$, chosen small, $V_{0, \varepsilon}$, that consists in the "low-frequency" and "high-frequency" parts of $U_{0}$ is also small in $H^{0, s}$. We have:

$$
\begin{aligned}
& \left\|V_{0, \varepsilon}\right\|_{\mathbf{H}^{0, s}}^{2}= \\
& =\int_{\mathbb{R}^{3}}\left(1+\left|\xi_{3}\right|^{2}\right)^{s}\left|\left\{1-\chi\left(\frac{|\xi|}{R_{\varepsilon}}\right)\left[1-\chi\left(\frac{\left|\xi_{3}\right|}{r_{\varepsilon}}\right)\left(1-\chi\left(\frac{\left|\xi_{h}\right|}{r_{\varepsilon}}\right)\right)\right]\right\} \widehat{U_{0}}(\xi)\right|^{2} d \xi \\
& \leq \int_{\left|\xi_{3}\right| \leq r_{\varepsilon}} \int_{\mathbb{R}_{\xi_{h}}^{2}}\left(1+\left|\xi_{3}\right|^{2}\right)^{s}\left|\widehat{U_{0}}(\xi)\right|^{2} d \xi_{h} d \xi_{3}+\int_{\left|\xi_{h}\right| \leq r_{\varepsilon}} \int_{\mathbb{R}_{\xi_{3}}}\left(1+\left|\xi_{3}\right|^{2}\right)^{s}\left|\widehat{U_{0}}(\xi)\right|^{2} d \xi_{3} d \xi_{h} \\
& \text { (5.6) } \quad+\int_{|\xi| \geq R_{\varepsilon}}\left(1+\left|\xi_{3}\right|^{2}\right)^{s}\left|\widehat{U_{0}}(\xi)\right|^{2} d \xi .
\end{aligned}
$$

First, we have

$$
\begin{aligned}
& \int_{\left|\xi_{3}\right| \leq r} \int_{\mathbb{R}_{\xi_{h}}^{2}}\left(1+\left|\xi_{3}\right|^{2}\right)^{s}\left|\widehat{U_{0}}(\xi)\right|^{2} d \xi_{h} d \xi_{3} \leq \\
& \leq \int_{\left|\xi_{3}\right| \leq r_{e}} \int_{\mathbb{R}_{\xi_{h}}^{2}}\left|\xi_{3}\right|^{2 \eta}\left[\left|\xi_{3}\right|^{-2 \eta}\left(1+\left|\xi_{3}\right|^{2}\right)^{s}\left|\widehat{U_{0}}(\xi)\right|^{2}\right] d \xi_{h} d \xi_{3} \leq C r_{\varepsilon}^{2 \eta}\left\|U_{0}\right\|_{L_{h}^{2} \dot{H}_{v}^{-2 \eta}}^{2} .
\end{aligned}
$$

Next,

$$
\begin{aligned}
& \int_{\left|\xi_{h}\right| \leq r} \int_{\mathbb{R}_{\xi_{3}}}\left(1+\left|\xi_{3}\right|^{2}\right)^{s}\left|\widehat{U_{0}}(\xi)\right|^{2} d \xi_{3} d \xi_{h} \leq \\
& \leq \int_{\left|\xi_{h}\right| \leq r} \int_{\mathbb{R}_{\xi_{3}}}\left|\xi_{h}\right|^{\eta}\left[\left|\xi_{h}\right|^{-\eta}\left(1+\left|\xi_{3}\right|^{2}\right)^{s}\left|\widehat{U_{0}}(\xi)\right|^{2}\right] d \xi_{3} d \xi_{h} \leq C r_{\varepsilon}^{2 \eta}\left\|U_{0}\right\|_{\dot{H}_{h}^{-\eta} H_{v}^{s}}^{2} .
\end{aligned}
$$

Finally, we have:

$$
\begin{aligned}
& \text { (5.9) } \int_{|\xi| \geq R_{\varepsilon}}\left(1+\left|\xi_{3}\right|^{2}\right)^{s}\left|\widehat{U_{0}}\right|^{2} d \xi \leq \\
& \leq C \int_{|\xi| \geq R_{\varepsilon}}\left(1+|\xi|^{2}\right)^{-\eta}\left(1+\left|\xi_{h}\right|^{2}\right)^{\eta}\left(1+\left|\xi_{3}\right|^{2}\right)^{s+\eta}\left|\widehat{U_{0}}\right|^{2} d \xi \leq C R_{\varepsilon}^{-2 \eta}\left\|U_{0}\right\|_{\mathbf{H}^{\eta, s+\eta}}^{2} .
\end{aligned}
$$


Let $\eta_{0}=\min (\eta, \gamma \eta)$. Summing (5.7)-(5.9) we obtain

$$
\left\|V_{0, \varepsilon}\right\|_{\mathbf{H}^{0, s}}^{2} \leq C\left(U_{0}\right) R_{\varepsilon}^{-2 \eta_{0}}
$$

Then, combining this and (5.5), we get:

$$
\left\|V_{\left.\varepsilon\right|_{t=0}}\right\|_{H^{0, s}} \leq C\left(U_{0}\right)\left(\varepsilon^{1+\alpha-2 \beta}+\varepsilon^{\beta \eta_{0}}\right) .
$$

\subsection{Proof of Theorem 5.1}

In the previous sections we obtained the following Strichartz estimates (see Lemma 5.3) which we only write here in the cases $p \in\{1,2\}$ : there exists a square summable sequence $c_{q}$ such that $\sum_{q} c_{q}^{2} \leq 1$ and for all $q$,

$$
\begin{aligned}
& \left\|\Delta_{q}^{v} \overline{U_{\varepsilon}}\right\|_{L^{1}\left(\mathbb{R}_{+}, L_{h, v}^{\infty, 2}\right)} \leq c_{q} 2^{-q s} C_{F, \rho} \varepsilon^{\frac{1-3 \alpha}{4}} R_{\varepsilon}^{\frac{7+9 \gamma}{2}}\left\|U_{0}\right\|_{H^{0, s}} . \\
& \left\|\Delta_{q}^{v} \overline{U_{\varepsilon}}\right\|_{L^{2}\left(\mathbb{R}_{+}, L_{h, v}^{\infty, 2}\right)} \leq c_{q} 2^{-q s} C_{F, \rho} \varepsilon^{\frac{1-3 \alpha}{8}} R_{\varepsilon}^{\frac{11+11 \gamma}{4}}\left\|U_{0}\right\|_{H^{0, s} .}
\end{aligned}
$$

and the initial data estimates (5.11):

$$
\left\|V_{\varepsilon \mid t=0}\right\|_{H^{0, s}} \leq C\left(U_{0}\right)\left(\varepsilon^{1+\alpha-2 \beta}+\varepsilon^{\beta \eta_{0}}\right) .
$$

In the following, athough it may vary from line to line, $d_{q}$ will denote a summable sequence, $c_{q}$ a square summable sequence and $C_{F, \rho, s, U_{0}}$ a constant depending on the listed coefficients.

Let us go back to system (5.3). The aim is to show that, if $\varepsilon$ is small enough, the solution $V_{\varepsilon}$ of this system is global in time. For this we apply the Leray projector $\mathbb{P}$, then truncate with $\Delta_{q}^{v}$, take the inner product in $L^{2}$ with $\Delta_{q}^{v} V_{\varepsilon}$ and integrate in time from zero to $t$ (remember that $\mathbb{P} V_{\varepsilon}=V_{\varepsilon}$ and $\left.\left(\mathbb{P} f \mid V_{\varepsilon}\right)_{L^{2}}=\left(f \mid V_{\varepsilon}\right)_{L^{2}}\right)$. We obtain the estimate:

$$
\begin{aligned}
\left\|\Delta_{q}^{v} V_{\varepsilon}(t)\right\|_{L^{2}}^{2}+2 \nu_{0, \varepsilon} & \int_{0}^{t}\left\|\Delta_{q}^{v} \nabla_{h} V_{\varepsilon}(\tau)\right\|_{L^{2}}^{2} d \tau \leq \\
& \leq\left\|\Delta_{q}^{v} V_{\varepsilon}(0)\right\|_{L^{2}}^{2}+\left(A_{1}\right)+\left(A_{2}\right)+\left(A_{3}\right)+\left(A_{4}\right),
\end{aligned}
$$

with

$$
\left\{\begin{array}{l}
A_{1}=\int_{0}^{t} \mid\left(\Delta_{q}^{v}\left(V_{\varepsilon} \cdot \nabla V_{\varepsilon}\right) \mid \Delta_{q}^{v} V_{\varepsilon}\right)_{L^{2}} d \tau \\
A_{2}=\int_{0}^{t} \mid\left(\Delta_{q}^{v}\left(V_{\varepsilon} \cdot \nabla \overline{U_{\varepsilon}}\right) \mid \Delta_{q}^{v} V_{\varepsilon}\right)_{L^{2}} d \tau \\
A_{3}=\int_{0}^{t} \mid\left(\Delta_{q}^{v}\left(\overline{U_{\varepsilon}} \cdot \nabla V_{\varepsilon}\right) \mid \Delta_{q}^{v} V_{\varepsilon}\right)_{L^{2}} d \tau \\
\left.A_{4}=\int_{0}^{t}\left|\left(\Delta_{q}^{v} \overline{U_{\varepsilon}} \cdot \nabla \overline{U_{\varepsilon}}\right)\right| \Delta_{q}^{v} V_{\varepsilon}\right)_{L^{2}} d \tau
\end{array} .\right.
$$

The first term is estimated in the usual way with Lemma 2.6 (see for example [15] for the proof): there exists a constant $C>0$ and a summable sequence $\left(d_{q}\right)_{q \in \mathbb{Z}}$ such that:

$$
\left|\left(\Delta_{q}^{v}\left(V_{\varepsilon} . \nabla V_{\varepsilon}\right) \mid \Delta_{q}^{v} V_{\varepsilon}\right)_{L^{2}}\right| \leq C d_{q} 2^{-2 q s}\left\|V_{\varepsilon}\right\|_{H^{0, s}}\left\|\nabla_{h} V_{\varepsilon}\right\|_{H^{0, s}}^{2},
$$


and from this we easily deduce that:

$$
A_{1} \leq C d_{q} 2^{-2 q s}\left\|V_{\varepsilon}\right\|_{\tilde{L}_{T}^{\infty} H^{0, s}}\left\|\nabla_{h} V_{\varepsilon}\right\|_{\tilde{L}_{T}^{2} H^{0, s}}^{2} .
$$

\subsubsection{An additional estimate}

As the viscosities are small as $\varepsilon$ goes to zero, we cannot use this estimate for $A_{2}, A_{3}$ or $A_{4}$ because of terms like $\left\|V_{\varepsilon}\right\|_{H^{0, s}}^{2}\left\|\nabla_{h} \overline{U_{\varepsilon}}\right\|_{H^{0, s}}^{2} / \nu_{\varepsilon}$, introduced in the energy and bootstrap methods. The only way for us is to take advantage of the norm $\left\|\overline{U_{\varepsilon}}\right\|_{L_{x_{h}, x_{3}}^{\infty, 2}}$ and the Strichartz estimates in order to balance this $1 / \nu_{\varepsilon}$. This is the aim of the following lemma (adapted from the one proven in [35], slightly different from [15]):

Lemma 5.4 For any $s>\frac{1}{2}$ there exists a constant $C=C\left(F, \rho, U_{0}, s\right)>0$ such that for all tempered distribution $w$ and all time $T>0$, we have:

$$
\begin{aligned}
& \int_{0}^{T}\left|\left(\Delta_{q}^{v}\left(w(t) \cdot \nabla \overline{U_{\varepsilon}}(t) \mid \Delta_{q}^{v} V_{\varepsilon}(t)\right)_{H^{0, s}}\right)\right| d t \leq \\
& \quad \leq C d_{q} 2^{-2 q s} \varepsilon^{\frac{1-3 \alpha}{4}} R_{\varepsilon}^{\frac{9}{2}(1+\gamma)}\left\|V_{\varepsilon}\right\|_{\tilde{L}_{T}^{\infty} H^{0, s}}\|w\|_{\tilde{L}_{T}^{\infty} H^{0, s}}
\end{aligned}
$$

where $\left(d_{q}\right)_{q \in\{-1\} \cup \mathbb{N}}$ is a positive summable sequence such that $\sum_{q} d_{q} \leq 1$ (and it depends on the three functions involved).

Proof. For the convenience of the reader we adapt here the proof given in [15] or [35]. Let us write, as in [15] or [35], the Bony decomposition into the following way (we refer to Definition 2.1):

$$
a . b=\sum_{q} S_{q-1}^{v} a . \Delta_{q}^{v} b+\sum_{q} S_{q+2}^{v} b . \Delta_{q}^{v} a .
$$

So we get the estimate:

$$
\left|\left(\Delta_{q}^{v}\left(w^{i} . \partial_{i} \overline{U_{\varepsilon}}\right) \mid \Delta_{q}^{v} V_{\varepsilon}\right)_{L^{2}}\right| \leq I_{1}+I_{2},
$$

with

$$
I_{1}=\left|\left(\Delta_{q}^{v}\left(\sum_{\left|q-q^{\prime}\right| \leq N_{0}} S_{q^{\prime}-1}^{v} w^{i} \cdot \Delta_{q^{\prime}}^{v} \partial_{i} \overline{U_{\varepsilon}}\right) \mid \Delta_{q}^{v} V_{\varepsilon}\right)_{L^{2}}\right|,
$$

and,

$$
I_{2}=\left|\left(\Delta_{q}^{v}\left(\sum_{q^{\prime} \geq q-N_{0}} S_{q^{\prime}+2}^{v} \partial_{i} \overline{U_{\varepsilon}} \cdot \Delta_{q^{\prime}}^{v} w^{i}\right) \mid \Delta_{q}^{v} V_{\varepsilon}\right)_{L^{2}}\right|,
$$

Using Hölder estimates we get:

$$
\int_{0}^{T} I_{1} d t \leq \sum_{\left|q-q^{\prime}\right| \leq N_{0}}\left\|S_{q^{\prime}-1}^{v} w^{i}\right\|_{L_{T}^{\infty} L_{h, v}^{2, \infty}}\left\|\Delta_{q^{\prime}}^{v} \partial_{i} \overline{U_{\varepsilon}}\right\|_{L_{T}^{1} L_{h, v}^{\infty, 2}}\left\|\Delta_{q}^{v} V_{\varepsilon}\right\|_{L_{T}^{\infty} L^{2}}
$$


We easily estimate the first term: using the Bernstein lemma (see 2.2), we obtain

$$
\left\|S_{q^{\prime}-1}^{v} w\right\|_{L_{T}^{\infty} L_{h, v}^{2, \infty}} \leq \sum_{q^{\prime \prime} \leq q^{\prime}-2}\left\|\Delta_{q^{\prime \prime}}^{v} w\right\|_{L_{T}^{\infty} L_{h, v}^{2, \infty}} \leq \sum_{q^{\prime \prime} \leq q^{\prime}-2} 2^{\frac{q^{\prime \prime}}{2}}\left\|\Delta_{q^{\prime \prime}}^{v} w\right\|_{L_{T}^{\infty} L^{2}},
$$

so, with the definition of Besov spaces there exists a square summable sequence $c_{q^{\prime \prime}}$ :

$$
\left\|S_{q^{\prime}-1}^{v} w\right\|_{L_{T}^{\infty} L_{h, v}^{2, \infty}} \leq \sum_{q^{\prime \prime} \leq q^{\prime}-2} 2^{q^{\prime \prime}\left(\frac{1}{2}-s\right)} c_{q^{\prime \prime}}\|w\|_{\tilde{L}_{T}^{\infty} H^{0, s}}
$$

As $s>\frac{1}{2}$ we get that:

$$
\left\|S_{q^{\prime}-1}^{v} w\right\|_{L_{T}^{\infty} L_{h, v}^{2, \infty}} \leq\left(\sum_{q^{\prime \prime} \leq q^{\prime}-2} 2^{2 q^{\prime \prime}\left(\frac{1}{2}-s\right)}\right)^{\frac{1}{2}}\left(\sum_{q^{\prime \prime} \leq q^{\prime}-2} c_{q^{\prime \prime}}^{2}\right)^{\frac{1}{2}}\|w\|_{\tilde{L}_{T}^{\infty} H^{0, s}} \leq C_{s}\|w\|_{\tilde{L}_{T}^{\infty} H^{0, s}} .
$$

The classical Bernstein lemma (recall that $\overline{U_{\varepsilon}}$ has frequencies localized in $\left.\mathcal{C}_{r_{\varepsilon}, R_{\varepsilon}}\right)$ and Strichartz estimates allow to write:

$$
\left\|\Delta_{q^{\prime}}^{v} \partial_{i} \overline{U_{\varepsilon}}\right\|_{L_{T}^{1} L_{h, v}^{\infty, 2}} \leq R_{\varepsilon}\left\|\Delta_{q^{\prime}}^{v} \overline{U_{\varepsilon}}\right\|_{L_{T}^{1} L_{h, v}^{\infty, 2}} \leq c_{q^{\prime}} 2^{-q^{\prime} s} C_{F, \rho} \varepsilon^{\frac{1-3 \alpha}{4}} R_{\varepsilon}^{\frac{9}{2}(1+\gamma)}\left\|U_{0}\right\|_{H^{0, s}} .
$$

The last term is estimated thanks to the Besov spaces definition. There exists a square summable sequence with $\sum_{q} c_{q}^{2} \leq 1$ such that:

$$
\left\|\Delta_{q}^{v} V_{\varepsilon}\right\|_{L_{T}^{\infty} L^{2}} \leq c_{q} 2^{-q s}\left\|V_{\varepsilon}\right\|_{\tilde{L}_{T}^{\infty} H^{0, s}}
$$

So going back to the estimate of (1) we obtain:

$$
\begin{aligned}
\int_{0}^{T} I_{1} d t \leq 2^{-2 q s} c_{q}\left(\sum_{\left|q-q^{\prime}\right| \leq N_{0}} c_{q^{\prime}} 2^{\left(q-q^{\prime}\right) s}\right) C_{F, \rho} \\
\varepsilon^{\frac{1-3 \alpha}{4}} R_{\varepsilon}^{\frac{9}{2}(1+\gamma)}\left\|U_{0}\right\|_{H^{0, s}}\left\|V_{\varepsilon}\right\|_{\tilde{L}_{T}^{\infty} H^{0, s}}\|w\|_{\tilde{L}_{T}^{\infty} H^{0, s}}
\end{aligned}
$$

where the term $\left(\sum_{\left|q-q^{\prime}\right| \leq N_{0}} c_{q^{\prime}} 2^{\left(q-q^{\prime}\right) s}\right)$ is nothing but the convolution of two sequences in $l^{1}(\mathbb{Z})$ and $l^{2}(\mathbb{Z})$, so it is in $l^{2}(\mathbb{Z})$ and

$$
\int_{0}^{T} I_{1} d t \leq C_{F, \rho, s, U_{0}} 2^{-2 q s} d_{q} \varepsilon^{\frac{1-3 \alpha}{4}} R_{\varepsilon}^{\frac{9}{2}(1+\gamma)}\left\|V_{\varepsilon}\right\|_{\tilde{L}_{T}^{\infty} H^{0, s}}\|w\|_{\tilde{L}_{T}^{\infty} H^{0, s}},
$$

For $I_{2}$, Hölder estimates imply:

$$
\int_{0}^{T} I_{2} d t \leq \sum_{q^{\prime} \geq q-N_{0}}\left\|S_{q^{\prime}+2}^{v} \partial_{i} \overline{U_{\varepsilon}}\right\|_{L_{T}^{1} L^{\infty}}\left\|\Delta_{q^{\prime}}^{v} w^{i}\right\|_{L_{T}^{\infty} L^{2}}\left\|\Delta_{q}^{v} V_{\varepsilon}\right\|_{L_{T}^{\infty} L^{2}}
$$


The first term is treated the same way as $I_{1}$ :

$$
\left\|S_{q^{\prime}+2}^{v} \partial_{i} \overline{U_{\varepsilon}}\right\|_{L_{T}^{1} L^{\infty}} \leq \sum_{q^{\prime \prime} \leq q^{\prime}+1}\left\|\Delta_{q^{\prime \prime}}^{v} \partial_{i} \overline{U_{\varepsilon}}\right\|_{L_{T}^{1} L^{2}} \leq \sum_{q^{\prime \prime} \leq q^{\prime}-2} R_{\varepsilon} 2^{\frac{q^{\prime \prime}}{2}}\left\|\Delta_{q^{\prime \prime}}^{v} \partial_{i} \overline{U_{\varepsilon}}\right\|_{L_{T}^{\infty} L_{h, v}^{\infty, 2}},
$$

Then, the very same arguments give that there exists a summable sequence $\left(d_{q}\right) \in l^{1}(\{-1\} \cup \mathbb{Z})$ such that:

$$
\int_{0}^{T} I_{2} d t \leq C_{F, \rho, s, U_{0}} 2^{-2 q s} d_{q} \varepsilon^{\frac{1-3 \alpha}{4}} R_{\varepsilon}^{\frac{9}{2}(1+\gamma)}\left\|V_{\varepsilon}\right\|_{\tilde{L}_{T}^{\infty} H^{0, s}}\|w\|_{\tilde{L}_{T}^{\infty} H^{0, s}} .
$$

The lemma is proven.

Going back to the energy estimate (5.12), this lemma allows us to obtain the existence of a summable sequence (once again denoted by $\left(d_{q}\right)_{q \in \mathbb{Z}}$ ) so that:

$$
A_{2}+A_{4} \leq C_{F, \rho, s, U_{0}} 2^{-2 q s} d_{q} \varepsilon^{\frac{1-3 \alpha}{4}} R_{\varepsilon}^{\frac{9}{2}(1+\gamma)}\left(\left\|V_{\varepsilon}\right\|_{\tilde{L}_{T}^{\infty} H^{0, s}}^{2}+\left\|V_{\varepsilon}\right\|_{\tilde{L}_{T}^{\infty} H^{0, s}}\left\|\overline{U_{\varepsilon}}\right\|_{\tilde{L}_{T}^{\infty} H^{0, s}}\right) .
$$

Unfortunately, this lemma is not useful for $A_{3}$ where the derivative is now applied to $V_{\varepsilon}$ which is not localized in frequency in $C_{r_{\varepsilon}, R_{\varepsilon}}$ as $\overline{U_{\varepsilon}}$. So using this lemma would force us to deal with an additionnal $2^{q}$ that would be impossible to absorb. This is the reason why we decompose it as in [15] or $[35]$ :

$$
\begin{gathered}
\left.A_{3}=\int_{0}^{T}\left|\left(\Delta_{q}^{v} \overline{U_{\varepsilon}} \cdot \nabla V_{\varepsilon}\right)\right| \Delta_{q}^{v} V_{\varepsilon}\right)_{L^{2}}\left|d t \leq \int_{0}^{T}\right|\left(\Delta_{q}^{v}\left({\overline{U_{\varepsilon}}}^{h} \cdot \nabla_{h} V_{\varepsilon}\right) \mid \Delta_{q}^{v} V_{\varepsilon}\right)_{L^{2}} \mid d t \\
+\int_{0}^{T}\left|\left(\Delta_{q}^{v}\left({\overline{U_{\varepsilon}}}^{3} \cdot \partial_{3} V_{\varepsilon}\right) \mid \Delta_{q}^{v} V_{\varepsilon}\right)_{L^{2}}\right| d t=A_{31}+A_{32}
\end{gathered}
$$

The term $A_{31}$ is dealt the same way as in the proof of Lemma 5.4 except that we take advantage of the estimate of $\left\|\nabla_{h} V_{\varepsilon}\right\|_{\tilde{L}_{T}^{2} H^{0, s}}$ (and therefore use the Strichartz estimate with $p=2$ ) and we obtain that:

$$
A_{31} \leq C_{F, \rho, s, U_{0}} 2^{-2 q s} d_{q} \varepsilon^{\frac{1-3 \alpha}{8}} R_{\varepsilon}^{\frac{11}{4}(1+\gamma)}\left\|V_{\varepsilon}\right\|_{\tilde{L}_{T}^{\infty} H^{0, s}}\left\|\nabla_{h} V_{\varepsilon}\right\|_{\tilde{L}_{T}^{2} H^{0, s}} .
$$

For the last term, as usual (we refer for example to [15]) we decompose:

$$
A_{32}=\int_{0}^{T}\left|\left(\Delta_{q}^{v}\left({\overline{U_{\varepsilon}}}^{3} \cdot \partial_{3} V_{\varepsilon}\right) \mid \Delta_{q}^{v} V_{\varepsilon}\right)_{L^{2}}\right| d t \leq A_{321}+A_{322},
$$

where

$$
\begin{aligned}
A_{321} & =\int_{0}^{T}\left|\left(\Delta_{q}^{v}\left(\sum_{\left|q-q^{\prime}\right| \leq N_{0}} S_{q^{\prime}-1}^{v}{\overline{U_{\varepsilon}}}^{3} \cdot \Delta_{q^{\prime}}^{v} \partial_{3} V_{\varepsilon}\right) \mid \Delta_{q}^{v} V_{\varepsilon}\right)_{L^{2}}\right| d t, \\
\text { and } A_{322} & =\int_{0}^{T}\left|\left(\Delta_{q}^{v}\left(\sum_{q^{\prime} \geq q-N_{0}} S_{q^{\prime}+2}^{v} \partial_{3} V_{\varepsilon} \cdot \Delta_{q^{\prime}}^{v} \bar{U}_{\varepsilon}^{3}\right) \mid \Delta_{q}^{v} V_{\varepsilon}\right)_{L^{2}}\right| d t
\end{aligned}
$$


Let us write that

$$
A_{322} \leq \sum_{q^{\prime} \geq q-N_{0}}\left\|S_{q^{\prime}+2}^{v} \partial_{3} V_{\varepsilon}\right\|_{L_{T}^{\infty} L_{h, v}^{2, \infty}}\left\|\Delta_{q^{\prime}}^{v} \bar{U}_{\varepsilon}^{3}\right\|_{L_{T}^{1} L_{h, v}^{\infty, 2}}\left\|\Delta_{q}^{v} V_{\varepsilon}\right\|_{L_{T}^{\infty} L^{2}} .
$$

The same arguments imply that for all $q^{\prime} \geq-1$,

$$
\left\|S_{q^{\prime}+2}^{v} \partial_{3} V_{\varepsilon}\right\|_{L_{T}^{\infty} L_{h, v}^{2, \infty}} \leq C 2^{q^{\prime}} \sum_{q^{\prime \prime} \leq q^{\prime}+1} 2^{\frac{q^{\prime \prime}}{2}}\left\|\Delta_{q^{\prime \prime}}^{v} V_{\varepsilon}\right\|_{L_{T}^{\infty} L^{2}} \leq 2^{q^{\prime}} C_{s}\left\|V_{\varepsilon}\right\|_{\tilde{L}_{T}^{\infty} H^{0, s}}
$$

And for all $q \geq 0$ we can absorb $2^{q^{\prime}}$ thanks to the anisotropic Bernstein lemma:

$$
\left\|\Delta_{q^{\prime}}^{v}{\overline{U_{\varepsilon}}}^{3}\right\|_{L_{T}^{1} L_{h, v}^{\infty, 2}} \leq 2^{-q^{\prime}}\left\|\Delta_{q^{\prime}}^{v} \partial_{3}{\overline{U_{\varepsilon}}}^{3}\right\|_{L_{T}^{1} L_{h, v}^{\infty, 2}} \leq 2^{-q^{\prime}} R_{\varepsilon}\left\|\Delta_{q^{\prime}}^{v}{\overline{U_{\varepsilon}}}^{3}\right\|_{L_{T}^{1} L_{h, v}^{\infty, 2},}
$$

which is also obviously true when $q^{\prime}=-1$ so using the Strichartz estimates and the same method as before we finally obtain:

$$
A_{322} \leq C_{F, \rho, s, U_{0}} 2^{-2 q s} d_{q} \varepsilon^{\frac{1-3 \alpha}{4}} R_{\varepsilon}^{\frac{9}{2}(1+\gamma)}\left\|V_{\varepsilon}\right\|_{\tilde{L}_{T}^{\infty} H^{0, s}}^{2}
$$

It is less easy to estimate $A_{321}$ because we cannot move the derivative, as we have done above, without introducing an additional $2^{q^{\prime}}$ (which couldn't be balanced by $R_{\varepsilon} 2^{-q^{\prime}}$ ). As in [15] or [35] we need to decompose $A_{321} \leq$ $A_{321}^{1}+A_{321}^{2}+A_{321}^{3}$ in the following way:

$$
\begin{aligned}
A_{321}^{1} & =\int_{0}^{T} \sum_{\left|q-q^{\prime}\right| \leq N_{0}}\left|\left(\left[\Delta_{q}^{v}, S_{q^{\prime}-1}^{v}{\overline{U_{\varepsilon}}}^{3}\right] \partial_{3} \Delta_{q^{\prime}}^{v} V_{\varepsilon} \mid \Delta_{q}^{v} V_{\varepsilon}\right)_{L^{2}}\right| d t, \\
A_{321}^{2} & =\int_{0}^{T} \sum_{\left|q-q^{\prime}\right| \leq N_{0}}\left|\left(\left(S_{q}^{v}-S_{q^{\prime}-1}^{v}\right){\overline{U_{\varepsilon}}}^{3} \partial_{3} \Delta_{q^{\prime}}^{v} V_{\varepsilon} \mid \Delta_{q}^{v} V_{\varepsilon}\right)_{L^{2}}\right| d t, \\
\text { and } \quad A_{321}^{3} & =\int_{0}^{T}\left|\left(S_{q}^{v}{\overline{U_{\varepsilon}}}^{3} \cdot \partial_{3} \Delta_{q}^{v} V_{\varepsilon} \mid \Delta_{q}^{v} V_{\varepsilon}\right)_{L^{2}}\right| d t
\end{aligned}
$$

The last term is dealt using an integration by parts:

$$
A_{321}^{3} \leq \frac{1}{2}\left\|S_{q}^{v} \partial_{3}{\overline{U_{\varepsilon}}}^{3}\right\|_{L_{T}^{1} L^{\infty}}\left\|\Delta_{q}^{v} V_{\varepsilon}\right\|_{L_{T}^{\infty} L^{2}}^{2} .
$$

The usual computations imply then:

$$
A_{321}^{3} \leq C_{F, \rho, s, U_{0}} 2^{-2 q s} d_{q} \varepsilon^{\frac{1-3 \alpha}{4}} R_{\varepsilon}^{\frac{9}{2}(1+\gamma)}\left\|V_{\varepsilon}\right\|_{\tilde{L}_{T}^{\infty} H^{0, s}}^{2} .
$$

For $A_{321}^{2}$ we use the same tools as in the proof of Lemma 5.4 together with the argument in the estimate of $A_{322}$. Then we use Lemma 2.6 (with $p=t=2$ 
and $r=\infty$ ) followed by the same arguments as above for $A_{321}^{1}$ and we obtain the estimate:

$$
A_{321} \leq C_{F, \rho, s, U_{0}} 2^{-2 q s} d_{q} \varepsilon^{\frac{1-3 \alpha}{4}} R_{\varepsilon}^{\frac{9}{2}(1+\gamma)}\left\|V_{\varepsilon}\right\|_{\tilde{L}_{T}^{\infty} H^{0, s}}^{2},
$$

which is the last part of the estimate of $A_{3}$ :

$$
\begin{gathered}
A_{3} \leq C_{F, \rho, s, U_{0}} 2^{-2 q s} d_{q} \varepsilon^{\frac{1-3 \alpha}{8}} R_{\varepsilon}^{\frac{11}{4}(1+\gamma)}\left\|V_{\varepsilon}\right\|_{\tilde{L}_{T}^{\infty} H^{0, s}}\left\|\nabla_{h} V_{\varepsilon}\right\|_{\tilde{L}_{T}^{2} H^{0, s}} . \\
+C_{F, \rho, s, U_{0}} 2^{-2 q s} d_{q} \varepsilon^{\frac{1-3 \alpha}{4}} R_{\varepsilon}^{\frac{9}{2}(1+\gamma)}\left\|V_{\varepsilon}\right\|_{\tilde{L}_{T}^{\infty} H^{0, s}}^{2}
\end{gathered}
$$

Plugging (5.13), (5.16) and (5.17) into (5.12) then multiplying by $2^{2 q s}$ before summing over $q$, we obtain, for all $T \in\left[0, T_{\varepsilon}^{*}[\right.$ :

$$
\begin{aligned}
\left\|V_{\varepsilon}\right\|_{\tilde{L}_{T}^{\infty} H^{0, s}}^{2} & +2 \nu_{0, \varepsilon}\left\|\nabla_{h} V_{\varepsilon}\right\|_{\tilde{L}_{T}^{2} H^{0, s}}^{2} \leq\left\|V_{\varepsilon}(0)\right\|_{H^{0, s}}^{2}+C\left\|V_{\varepsilon}\right\|_{\tilde{L}_{T}^{\infty} H^{0, s}}\left\|\nabla_{h} V_{\varepsilon}\right\|_{\tilde{L}_{T}^{2} H^{0, s}}^{2} \\
& +C_{F, \rho, s, U_{0}} \varepsilon^{\frac{1-3 \alpha}{4}} R_{\varepsilon}^{\frac{9}{2}(1+\gamma)}\left(\left\|V_{\varepsilon}\right\|_{\tilde{L}_{T}^{\infty} H^{0, s}}^{2}+\left\|V_{\varepsilon}\right\|_{\tilde{L}_{T}^{\infty} H^{0, s}} \|{\overline{U_{\varepsilon}}}_{\tilde{L}_{T}^{\infty} H^{0, s}}\right) \\
& +C_{F, \rho, s, U_{0}} \varepsilon^{\frac{1-3 \alpha}{8}} R_{\varepsilon}^{\frac{11}{4}(1+\gamma)}\left\|V_{\varepsilon}\right\|_{\tilde{L}_{T}^{\infty} H^{0, s}}\left\|\nabla_{h} V_{\varepsilon}\right\|_{\tilde{L}_{T}^{2} H^{0, s}}
\end{aligned}
$$

The classical estimate $a b \leq \frac{a^{2}+b^{2}}{2}$ allows us to write:

$$
\begin{aligned}
C_{F, \rho, s, U_{0}} \varepsilon^{\frac{1-3 \alpha}{8}} R_{\varepsilon}^{\frac{11}{4}(1+\gamma)}\left\|V_{\varepsilon}\right\|_{\tilde{L}_{T}^{\infty} H^{0, s}}\left\|\nabla_{h} V_{\varepsilon}\right\|_{\tilde{L}_{T}^{2} H^{0, s}} \leq \\
\quad \leq \nu_{0, \varepsilon}\left\|\nabla_{h} V_{\varepsilon}\right\|_{\tilde{L}_{T}^{2} H^{0, s}}^{2}+\frac{C_{F, \rho, s, U_{0}}}{\nu_{0, \varepsilon}} \varepsilon^{\frac{1-3 \alpha}{4}} R_{\varepsilon}^{\frac{11}{2}(1+\gamma)}\left\|V_{\varepsilon}\right\|_{\tilde{L}_{T}^{\infty} H^{0, s}}^{2}
\end{aligned}
$$

Remark 5.5 It is important to outline that it is here that Strichartz estimates balance $1 / \nu_{\varepsilon, 0}$.

On the other hand, energy estimates in $H^{0, s}$ for system (5.2) and Proposition 3.1 imply that (if $\varepsilon$ is small enough):

$$
\begin{aligned}
\left\|\overline{U_{\varepsilon}}(t)\right\|_{H^{0, s}} \leq\left\|\overline{U_{\varepsilon}}(0)\right\|_{H^{0, s}} & \leq\left\|\left(I_{d}-\mathbb{P}_{2}\right) \overline{U_{\varepsilon}}(t)\right\|_{H^{0, s}} \\
& \leq\left(1+C_{F}\left|\nu_{\varepsilon}-\nu_{\varepsilon}^{\prime}\right| \varepsilon R_{\varepsilon}^{2}\right)\left\|U_{0}\right\|_{H^{0, s}} \leq 2\left\|U_{0}\right\|_{H^{0, s}} .
\end{aligned}
$$

Then we can write:

$$
C_{F, \rho, s, U_{0}}\left(\left\|V_{\varepsilon}\right\|_{\tilde{L}_{T}^{\infty} H^{0, s}}^{2}+\left\|V_{\varepsilon}\right\|_{\tilde{L}_{T}^{\infty} H^{0, s}} \|{\overline{U_{\varepsilon}}}_{\tilde{L}_{T}^{\infty} H^{0, s}}\right) \leq C_{F, \rho, s, U_{0}}\left(1+\left\|V_{\varepsilon}\right\|_{\tilde{L}_{T}^{\infty} H^{0, s}}^{2}\right) .
$$

Replacing $R_{\varepsilon}$ by its value and using (5.11) we finally get:

$$
\begin{aligned}
& \left\|V_{\varepsilon}\right\|_{\tilde{L}_{T}^{\infty} H^{0, s}}^{2}+\nu_{0, \varepsilon}\left\|\nabla_{h} V_{\varepsilon}\right\|_{\tilde{L}_{T}^{2} H^{0, s}}^{2} \\
& \quad \leq C\left(\varepsilon^{\beta \eta_{0}}+\varepsilon^{1+\alpha-2 \beta}\right)^{2}+C\left\|V_{\varepsilon}\right\|_{\tilde{L}_{T}^{\infty} H^{0, s}}\left\|\nabla_{h} V_{\varepsilon}\right\|_{\tilde{L}_{T}^{2} H^{0, s}}^{2} \\
& \quad+C_{F, \rho, s, U_{0}} \varepsilon^{\frac{1-3 \alpha}{4}-\frac{9}{2} \beta(1+\gamma)}+\Phi(\varepsilon)\left\|V_{\varepsilon}\right\|_{\tilde{L}_{T}^{\infty} H^{0, s}}^{2},
\end{aligned}
$$


where

$$
\Phi(\varepsilon)=C_{F, \rho, s, U_{0}}\left(\varepsilon^{\frac{1-3 \alpha}{4}-\frac{9}{2} \beta(1+\gamma)}+\varepsilon^{\frac{1-7 \alpha}{4}-\frac{11}{2} \beta(1+\gamma)}\right) .
$$

In order to set up the usual boostrap argument let us define:

$$
T_{\varepsilon}=\sup \{t \in] 0, T_{\varepsilon}^{*}\left[\quad \text { such that }\left\|V_{\varepsilon}\right\|_{\tilde{L}_{t}^{\infty} H^{0, s}} \leq \frac{\nu_{0, \varepsilon}}{2 C}\right\} .
$$

We are sure that $T_{\varepsilon}>0$ as soon as:

$$
\left\|V_{\varepsilon}(0)\right\|_{H^{0, s}} \leq C\left(\varepsilon^{\beta \eta_{0}}+\varepsilon^{1+\alpha-2 \beta}\right) \leq \frac{\nu_{0, \varepsilon}}{4 C},
$$

that is (when $\varepsilon$ is small enough) when (recall that $\nu_{0, \varepsilon}=\min (1, \rho) \varepsilon^{\alpha}$ ):

$$
\left\{\begin{array}{l}
\beta \eta_{0}>\alpha \\
1+\alpha-2 \beta>\alpha
\end{array}\right.
$$

For all $T<T_{\varepsilon}$,

$$
\begin{aligned}
\left\|V_{\varepsilon}\right\|_{\tilde{L}_{T}^{\infty} H^{0, s}}^{2}+\frac{\nu_{0, \varepsilon}}{2} \| \nabla_{h} V_{\varepsilon} & \|_{\tilde{L}_{T}^{2} H^{0, s}}^{2} \leq C\left(\varepsilon^{\beta \eta_{0}}+\varepsilon^{1+\alpha-2 \beta}\right)^{2} \\
& +C_{F, \rho, s, U_{0}} \varepsilon^{\frac{1-3 \alpha}{4}-\frac{9}{2} \beta(1+\gamma)}+\Phi(\varepsilon)\left\|V_{\varepsilon}\right\|_{\tilde{L}_{T}^{\infty} H^{0, s}}^{2} .
\end{aligned}
$$

To be able to conclude we need to adjust the parameters: in order to complete the boostrap argument, we need the following estimates:

$$
\left\{\begin{array}{l}
\Phi(\varepsilon) \leq \frac{1}{2} \\
C_{F, \rho, s, U_{0}} \varepsilon^{\frac{1-3 \alpha}{4}-\frac{9}{2} \beta(1+\gamma)}+C\left(\varepsilon^{\beta \eta_{0}}+\varepsilon^{1+\alpha-2 \beta}\right)^{2} \leq\left(\frac{\varepsilon^{\alpha}}{4 C}\right)^{2}
\end{array}\right.
$$

As $\varepsilon$ goes to zero, it is sufficient to require:

$$
\left\{\begin{array}{l}
\frac{\alpha}{\eta_{0}}<\beta<\frac{1}{2} \\
\frac{1-11 \alpha}{4}-\frac{11}{2} \beta(1+\gamma)>0
\end{array}\right.
$$

We refer to the last section for a discussion on the parameters. For now let us just say that if $\alpha<\frac{1}{11}$ we can choose parameters $\beta$ and $\gamma$ satisfying (5.21). Plugging it into the energy estimates allows us to write that for all $T<T_{\varepsilon}$,

$$
\left\|V_{\varepsilon}\right\|_{\tilde{L}_{T}^{\infty} H^{0, s}}^{2}+\nu_{0, \varepsilon}\left\|\nabla_{h} V_{\varepsilon}\right\|_{\tilde{L}_{T}^{2} H^{0, s}}^{2} \leq \frac{\varepsilon^{2 \alpha}}{8 C} \leq \frac{1}{2}\left(\frac{\nu_{0}}{2 C}\right)^{2} .
$$

And then by contradiction it gives us that $T_{\varepsilon}=T_{\varepsilon}^{*}=\infty$ and $V_{\varepsilon}$ and the solution $U_{\varepsilon}$ are global in time. Moreover $U_{\varepsilon}$ goes to zero in the sense that the energy estimate (5.22) for $V_{\varepsilon}=U_{\varepsilon}-\overline{U_{\varepsilon}}$ is bounded by a positive power of $\varepsilon$ and goes to zero. And, with this classical bootstrap argument, we have proven the second part of Theorem 5.1: the solution $U_{\varepsilon}$ goes to zero in a certain way. 
Remark 5.6 We have shown that the solution $U_{\varepsilon}$ goes to zero in a certain way but we didn't mention anywhere the quasi-geostrophic system. Indeed the solution of the anisotropic quasi-geostrophic system with zero initial data is zero. That is also the reason why (contrary to [8]) we didn't have to use the orthogonal projectors on oscillating or quasi-geostrophic vector fields.

Remark 5.7 Technical problems forced $\alpha<\frac{1}{11}$ but the question of the optimal value of $\alpha$ is open (see the next paragraph).

\subsection{Discussion on the parameter $\alpha$}

In the previous section we concluded the boostrap argument thanks to a precise estimation of the parameters $\beta$ and $\gamma(\alpha, \eta$ and $p$ being given in the beginning). In order to conclude the classical boostrap method, we required that the parameters satisfy (5.20). It is sufficient to have:

$$
\left\{\begin{array}{l}
\frac{\alpha}{\eta_{0}}<\beta<\frac{1}{2} \\
\frac{1-3 \alpha}{4}-\frac{9}{2} \beta(1+\gamma)>2 \alpha \\
\frac{1-3 \alpha}{4}-\frac{9}{2} \beta(1+\gamma)>0 \\
\frac{1-7 \alpha}{4}-\frac{11}{2} \beta(1+\gamma)>0
\end{array}\right.
$$

which is implied by:

$$
\left\{\begin{array}{l}
\frac{\alpha}{\eta_{0}}<\beta<\frac{1}{2} \\
\frac{1-11 \alpha}{4}-\frac{11}{2} \beta(1+\gamma)>0
\end{array}\right.
$$

Choosing $\gamma=1$, so $\eta_{0}=\eta$ and the problem is then, given $\alpha>0$ and $\eta>0$, to find $\beta$ so that:

$$
\left\{\begin{array}{l}
\frac{\alpha}{\eta}<\beta<\frac{1}{2} \\
1>11 \alpha+44 \beta .
\end{array}\right.
$$

Choosing $\eta>0$ large enough and $\beta>0$ such that $\alpha<\beta \eta$, simple calculations imply

$$
\alpha<\frac{1}{11+\frac{44}{\eta}} .
$$

The greatest possible value for the parameter $\alpha$ is $\frac{1}{11}$ and the greatest we want to choose $\alpha$ (nearest $\alpha<\frac{1}{11}$ ), the greatest has to be $\eta$ which means we must require more regularity on the initial data. Typically, choose $\alpha<\frac{1}{11}$, then choose $\eta>0$ such that $\alpha<\frac{1}{11+\frac{44}{\eta}}$, which implies that $\frac{\alpha}{\eta}<\frac{1-11 \alpha}{44}$. Finally, use (5.23) and choose $\beta$ between those two values $(\gamma=1)$. 


\section{References}

[1] Babin, A., Mahalov, A. And Nicolaenko, B.: Global splitting, integrability and regularity of 3D Euler and Navier-Stokes equations for uniformly rotating fluids. European J. Mech. B Fluids 15 (1996), no. 3, 291-300.

[2] Babin, A., Mahalov, A. And Nicolaenko, B.: Global regularity of 3D rotating Navier-Stokes equations for resonant domains. Indiana Univ. Math. J. 48 (1999), no. 3, 1133-1176.

[3] Babin, A., Mahalov, A. And Nicolaenko, B.: Strongly stratified limit of 3D primitive equations in an infinite layer. In Advances in wave interaction and turbulence (South Hadley, MA, 2000), 1-11. Contemp. Math. 283. Amer. Math. Soc., Providence, RI, 2001.

[4] Beale, T. and Bourgeois, A.: Validity of the quasigeostrophic model for large-scale flow in the atmosphere and ocean. SIAM J. Math. Anal. 25 (1994), no. 4, 1023-1068.

[5] Bony, J.-M.: Calcul symbolique et propagation des singularités pour les équations aux dérivées partielles non linéaires. Ann. Sci. École Norm. Sup. (4) 14 (1981), no. 2, 209-246.

[6] Bougeault, P. and Sadourny, R.: Dynamique de l'atmosphère et de l'océan. Editions de l'Ecole Polytechnique, 2001.

[7] Charve, F.: Global well-posedness and asymptotics for a geophysical fluid system. Comm. Partial Differential Equations 29 (2004), no. 11-12, 1919-1940.

[8] Charve, F.: Convergence of weak solutions for the primitive system of the quasigeostrophic equations. Asymptot. Anal. 42 (2005), no. 3-4, 173-209.

[9] Charve, F.: Asymptotics and vortex patches for the quasigeostrophic approximation. J. Math. Pures Appl. 85 (2006), no. 4, 493-539.

[10] Charve, F.: Global well-posedness for the primitive equations with less regular initial data. Ann. Fac. Sci. Toulouse Math. 17 (2008), no. 2, 221-238.

[11] Chemin, J.-Y.: Remarques sur l'existence globale pour le système de Navier-Stokes incompressible. SIAM J. Math. Anal. 23 (1992), no. 1, 20-28.

[12] Chemin, J.-Y.: Fluides parfaits incompressibles. Astérisque 230, 1995.

[13] Chemin, J.-Y.: A propos d'un problème de pénalisation de type antisymétrique. J. Math. Pures Appl. 76 (1997), no. 9, 739-755.

[14] Chemin, J.-Y., Desjardins, B., Gallagher, I. and Grenier, E.: Anisotropy and dispersion in rotating fluids. In Nonlinear Partial Differential Equations and their application, Collège de France Seminar, 171-192. Stud. Math. Appl. 31. North-Holland, Amsterdam, 2002.

[15] Chemin, J.-Y., Desjardins, B., Gallagher, I. and Grenier, E.: Fluids with anisotropic viscosity. M2AN Math. Model. Numer. Anal. 34 (2000), no. 2, 315-335. 
[16] Chemin, J.-Y., Desjardins, B., Gallagher, I. and Grenier, E.: Ekman boundary layers in rotating fluids ESAIM Controle Optim. Calc. Var. 8 (2002), 441-466.

[17] Chemin, J.-Y., Desjardins, B., Gallagher, I. and Grenier, E.: Mathematical Geophysics. An introduction to rotating fluids and the Navier-Stokes equations. Oxford Lecture Series in Mathematics and its Applications 32. The Claredon Press, Oxford University Press, Oxford, 2006.

[18] Cushman-Roisin, B.: Introduction to geophysical fluid dynamics. Prentice-Hall, 1994.

[19] Dutrifoy, A.: Slow convergence to vortex patches in quasigeostrophic balance. Arch. Ration. Mech. Anal. 171 (2004), no. 3, 417-449.

[20] Dutrifoy, A.: Examples of dispersive effects in non-viscous rotating fluids J. Math. Pures Appl. 84 (2005), no. 3, 331-356.

[21] Embid, P. ANd Majda, A.: Averaging over fast gravity waves for geophysical flows with arbitrary potential vorticity. Comm. Partial Differential Equations 21 (1996), no. 3-4, 619-658.

[22] Embid, P. ANd MajdA, A.: Averaging over fast gravity waves for geophysical flows with unbalanced initial data. Theoretical and computational fluid dynamics 11 (1998), 155-169.

[23] Fujita, H. And Kato, T.: On the Navier-Stokes initial value problem I. Arch. Rational Mech. Anal. 16 (1964), 269-315.

[24] Gallagher, I.: Applications of Schochet's Methods to Parabolic Equation. J. Math. Pures Appl. 77 (1998), no. 10, 989-1054.

[25] Greenspan, H. P.: The theory of rotating fluids. Cambridge Monographs on Mechanics and Applied Math. Cambrigde Univ. Press, Cambridge, 1980.

[26] Grenier, E. And Masmoudi, N.: Ekman layers of rotating fluid, the case of well prepared initial data. Comm. Partial Differential Equations 22 (1997), no. 5-6, 953-975.

[27] Hörmander, L.: Linear partial differential operators. Die Grundlehren der mathematischen Wissenschaften 116. Springer-Verlag, New York, 1969.

[28] Iftimie, D.: The resolution of the Navier-Stokes equations in anisotropic spaces. Rev. Mat. Iberoamericana 15 (1999), no. 1, 1-36.

[29] Iftimie, D.: Approximation of the quasigeostrophic system with the primitive systems. Asymptot. Anal. 21 (1999), no. 2, 89-97.

[30] Iftimie, D.: A uniqueness result for the Navier-Stokes equations with vanishing vertical viscosity. SIAM J. Math. Anal. 33 (2002), no. 6, 1483-1493.

[31] Leray, J.: Essai sur le mouvement d'un liquide visqueux emplissant l'espace. Acta Matematica 63 (1933), 193-248.

[32] Lions, J.-L., Temam, R. And Wang, S.: New formulations of the primitive equations of atmosphere and applications. Nonlinearity $\mathbf{5}$ (1992), no. 2, $237-288$. 
[33] Lions, J.-L., Temam, R. And Wang, S.: Geostrophic asymptotics of the primitive equations of the atmosphere. Topol. Methods Nonlinear Anal. 4 (1994), no. 2, 253-287.

[34] Lions, J.-L.: Mathematical Topics in Fluid Mechanics, Vol. I, Incompressible Models. Oxford Lecture Series in Mathematics and its Applications 3. The Clarendon Press, Oxford University Press, New York, 1996.

[35] NGo, V.-S.: Rotating Fluids with small viscosity Int. Math. Res. Not. IMRN (2009), no. 10, 1860-1890.

[36] PAicu, M.: Étude asymptotique pour les fluides anisotropes en rotation rapide dans le cas périodique. J. Math. Pures Appl. 83 (2004), no. 2, 163-242.

[37] Paicu, M.: Équation périodique de Navier-Stokes sans viscosité dans une direction. Comm. Partial Differential Equations 30 (2005), no. 7-9, 1107-1140.

[38] PAICU, M.: Équation anisotrope de Navier-Stokes dans des espaces critiques. Rev. Mat. Iberoamericana 21 (2005), no. 1, 179-235.

[39] Pedlosky, J.: Geophysical fluid dynamics. Springer, 1979.

[40] Sablé-Tougeron, M.: Régularité microlocale pour des problèmes aux limites non linéaires. Ann. Inst. Fourier 36 (1986), no. 1, 39-82.

Recibido: 30 de junio de 2009

Frédéric Charve

Université Paris-Est Créteil (UPEC)

Laboratoire d'Analyse et de Mathématiques Appliquées (UMR 8050)

61 Avenue du Général de Gaulle 94010 Créteil Cedex, France. frederic.charve@univ-paris12.fr

Van-Sang Ngo

Université Paris-Est Créteil (UPEC)

Laboratoire d'Analyse et de Mathématiques Appliquées (UMR 8050)

61 Avenue du Général de Gaulle

94010 Créteil Cedex, France.

Van-Sang. Ngo@univ-paris12.fr

This work was partially supported by the ANR ("Agence Nationale de la Recherche", reference ANR-08-BLAN-0301-01). 